ARTICLE

Received 11 Apr 2016 | Accepted 20 Oct 2016 | Published 29 Nov 2016

DOI: $10.1038 /$ ncomms 13650

OPEN

\title{
PI3 kinase inhibition improves vascular malformations in mouse models of hereditary haemorrhagic telangiectasia
}

Roxana Ola ${ }^{1}$, Alexandre Dubrac ${ }^{1}$, Jinah Han ${ }^{1}$, Feng Zhang ${ }^{1}$, Jennifer S. Fang ${ }^{1}$, Bruno Larrivée ${ }^{1} \dagger$, Monica Lee ${ }^{2}$, Ana A. Urarte ${ }^{3}$, Jan R. Kraehling ${ }^{2}$, Gael Genet ${ }^{1}$, Karen K. Hirschi ${ }^{1}$, William C. Sessa ${ }^{2}$, Francesc V. Canals ${ }^{4}$, Mariona Graupera ${ }^{3}$, Minhong Yan ${ }^{5}$, Lawrence H. Young ${ }^{1}$, Paul S. Oh ${ }^{6}$ \& Anne Eichmann $1,7,8$

Activin receptor-like kinase 1 (ALK1) is an endothelial serine-threonine kinase receptor for bone morphogenetic proteins (BMPs) 9 and 10. Inactivating mutations in the ALK1 gene cause hereditary haemorrhagic telangiectasia type 2 (HHT2), a disabling disease characterized by excessive angiogenesis with arteriovenous malformations (AVMs). Here we show that inducible, endothelial-specific homozygous Alk1 inactivation and BMP9/10 ligand blockade both lead to AVM formation in postnatal retinal vessels and internal organs including the gastrointestinal (GI) tract in mice. VEGF and PI3K/AKT signalling are increased on Alk1 deletion and BMP9/10 ligand blockade. Genetic deletion of the signal-transducing Vegfr2 receptor prevents excessive angiogenesis but does not fully revert AVM formation. In contrast, pharmacological PI3K inhibition efficiently prevents AVM formation and reverts established AVMs. Thus, Alk1 deletion leads to increased endothelial PI3K pathway activation that may be a novel target for the treatment of vascular lesions in HHT2.

\footnotetext{
${ }^{1}$ Cardiovascular Research Center, Department of Internal Medicine, Yale University School of Medicine, New Haven, Connecticut 06511, USA. ${ }^{2}$ Department of Pharmacology, Yale University School of Medicine, New Haven, Connecticut 06520, USA. ${ }^{3}$ Vascular Signalling Laboratory, Institut d'Investigació Biomèdica de Bellvitge, L'Hospitalet de Llobregat, Barcelona 08908, Spain. ${ }^{4}$ Translation Research Laboratory, Catalan Institute of Oncology, Idibell, Barcelona 08908, Spain. ${ }^{5}$ Molecular Oncology, Genentech, Inc., South San Francisco, California 94080-4990, USA. ${ }^{\circ}$ Department of Physiology and Functional Genomics, University of Florida College of Medicine, PO Box 100274, Gainesville, Florida 32610, USA. ${ }^{7}$ Department of Cellular and Molecular Physiology, Yale University School of Medicine, New Haven, Connecticut 06520, USA. ${ }^{8}$ Inserm U970, Paris Cardiovascular Research Center, Paris 75015, France. $\dagger$ Present address: Department of Ophthalmology, Maisonneuve-Rosemont Hospital, 21 Research Centre, University of Montreal, Montreal, QC, Canada H1T 2M4. Correspondence and requests for materials should be addressed to A.E. (email: anne.eichmann@yale.edu).
} 
aemorrhagic telangiectasia (HHT) is an autosomaldominant inherited vascular disorder that affects $\sim 1$ in 5,000 people. Patients develop multiple focal vascular malformations including capillary telangiectasies and arteriovenous malformations (AVMs) ${ }^{1}$. These lesions are fragile and prone to bleeding, and large calibre AVMs cause pulmonary and systemic shunting that can be physiologically significant ${ }^{1}$. More than $95 \%$ of HHT cases are caused by mutations in transforming growth factor- $\beta$ (TGF- $\beta$ )/bone morphogenetic protein (BMP) signalling pathway genes, including the surface receptors Endoglin (ENG, mutated in HHT1) and ACVRL1 (hereafter referred as $A L K 1$, mutated in HHT2), and the signalling pathway effector SMAD4-juvenile polyposis ${ }^{2-5}$. ENG and ALK1 proteins are expressed predominantly at the surface of endothelial cells, where they bind members of the TGF- $\beta$ /BMP family, including TGF- $\beta$, BMP9 and BMP10 (refs 6-8). ENG acts as an auxiliary co-receptor that promotes signalling through ALK1 (ref. 9). Ligand binding activates cytoplasmic regulatory SMADs 1, 5 and 8 , which subsequently complex with SMAD4 and translocate into the nucleus to regulate gene expression ${ }^{7}$. Thus, HHT is caused by mutations leading to deregulation in this signalling pathway, but how these mutations induce vascular malformations remains unclear. Identifying such mechanisms could provide novel approaches to prevent vascular malformations in HHT patients.

Mouse models have provided insights into Eng and Alk1 function. Heterozygous mutations in either gene give rise to vascular lesions, but these form at low frequency and late in life, making them inconvenient to study ${ }^{10,11}$. Homozygous global $E_{n}{ }^{10}$ and $A l k 1$ gene inactivation in mice ${ }^{6}$ and zebrafish ${ }^{12}$ leads to embryonic death due to AVMs, again rendering the study of molecular mechanisms difficult. However, postnatal tamoxifen (Tx)-inducible, endothelial-specific homozygous deletion of either gene combined with angiogenic or pro-inflammatory stimuli induces HHT-like vascular malformations including excessive angiogenesis, enlarged veins and $\mathrm{AVMs}^{13-16}$. Thus, loss of both copies of endothelial Alk1 or Eng genes in the context of active angiogenesis is thought to engender vascular lesions.

Here we investigate AVM formation in the retina and gastrointestinal (GI) tract using $C d h 5-C r e^{E R T 2}$ mice $^{17}$ crossed with $A l k 1^{\mathrm{fl} / \mathrm{fl}}\left(A l k 1^{i \Delta E C}\right)$ mice $\mathrm{in}^{14,18}$ in which endothelial-specific homozygous deletion of $A l k 1$ can be induced postnatally with Tx and blocking antibodies (blABs) against the BMP9/10 ligands ${ }^{19}$. We show that blocking BMP9-ALK1 signalling enhances pro-angiogenic signalling induced by vascular endothelial growth factor (VEGF), the major angiogenic growth factor known to date ${ }^{20}$, but also enhances phosphatidyl inositol 3-kinase (PI3K)-AKT signalling independently of exogenous VEGF. We show that targeting Vegfr2 prevents angiogenesis in $A l k 1^{i \Delta E C}$ mice but does not rescue normal vascular patterning and AVM formation, whereas PI3K inhibition rescues vascular malformations in BMP signalling-deficient retinas and GI tract, identifying PI3K pathway inhibition as a novel putative treatment approach for HHT patients.

\section{Results}

Blocking BMP9/10 signalling leads to vascular malformations. We used postnatal day 5 (P5) Cdh5-Cre $e^{E R T 2} ; A l k 1^{\mathrm{fl} / \mathrm{fl}}$ mice $\left(A l k 1^{i \Delta E C}\right)$ treated with a single dose of $50 \mu \mathrm{g} \mathrm{Tx}$ at $\mathrm{P} 3$ and mice treated with $\mathrm{BMP} / 10 \mathrm{blAB}^{19}\left(10 \mathrm{mg} \mathrm{kg}^{-1}\right.$, intraperitoneal (i.p.)) between P2 and P4 (Fig. 1a and Supplementary Fig. 1a). Tx-injected Cre-negative littermates and uninjected wild-type (no antibody) littermates were used as controls. Efficient Alk1 gene deletion was verified by quantitative PCR (qPCR) from mouse lung endothelial cells (mLECs; Supplementary Fig. 1b) and loss of endothelial staining with an anti-Alk1 antibody (Supplementary Fig. 1c,d). BMP9/10 blockade did not affect Alk1 expression (Supplementary Fig. 1c,e).

At P5, the morphology of retinal and organ vasculature was analysed by injection of latex dye (Fig. 1). Control retinas and the a

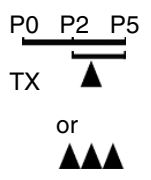

BMP9/10 bIAB

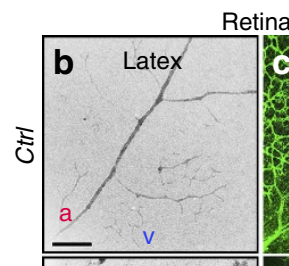

Retinas
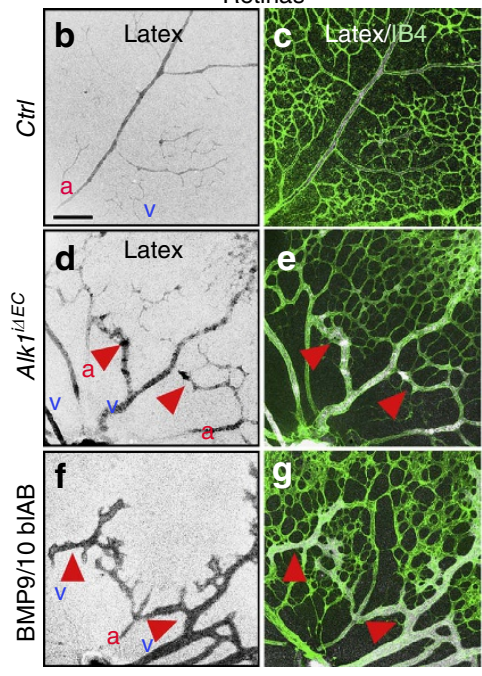
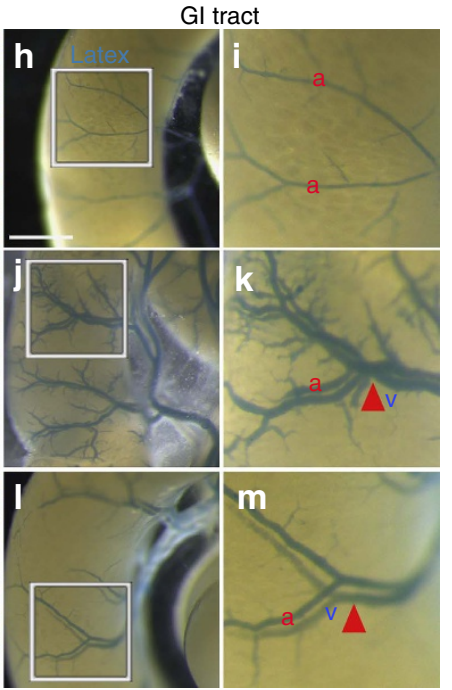
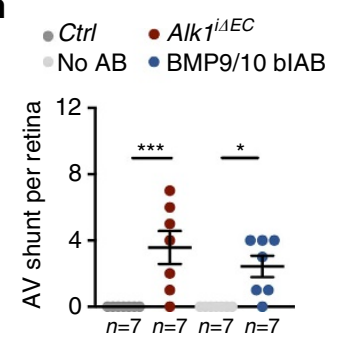

$\mathbf{0}$

Ctrl $\quad A l k 1^{i \Delta E C}$

No $A B \cdot B M P 9 / 10$ bIAB

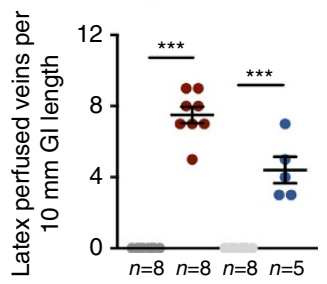

Figure 1 | Blocking BMP9/10-Alk1 signalling induces vascular malformations. (a) Schematic representation of the experimental strategy used to delete Alk1 or to block BMP9/10 ligands (PO-P5). Arrowheads indicate intra-gastric injection of $50 \mu \mathrm{g}$ Tx at postnatal day 3 (P3) and i.p. administration of BMP9/ $10 \mathrm{blAB},\left(10 \mathrm{mg} \mathrm{kg}^{-1}\right)$ at P2-P4. (b-g) P5 mouse retinas containing latex dye injected into the left ventricle of the heart. Vascular staining with latex dye (black in single images in $\mathbf{b}, \mathbf{d}, \mathbf{f}$ and white in merged images in $\mathbf{c}, \mathbf{e}, \mathbf{g})$ and IB4, (green) in $\mathbf{c}, \mathbf{e}, \mathbf{g}$ of retinal flat mounts from control (Ctrl) $(\mathbf{b}, \mathbf{c}), A l k 7^{i \Delta E C}$ (d,e) and BMP9/10 blAB-injected $(\mathbf{f}, \mathbf{g})$ mice. Red arrowheads indicate retinal AVMs. (h-m) Vascular staining with latex dye of small intestine (blue) in Ctrl (h,i), $A / k 7^{i \Delta E C}(\mathbf{j}, \mathbf{k})$ and BMP9/10 bIAB-injected $(\mathbf{I}, \mathbf{m})$ P5 mice. $\mathbf{i}, \mathbf{k}, \mathbf{m}$ are magnified areas of boxed areas in $\mathbf{h}, \mathbf{j}, \mathbf{l}$. Red arrowheads indicate latex-perfused veins. (n) Quantification of AV shunt number in the retinas. Each dot represents a retina. $n=7$ Retinas per group. Error bars: s.e.m., ${ }^{\star} P<0.05$ and ${ }^{\star \star \star} P<0.001$, Mann-Whitney U-test. (o) Quantification of latex-perfused veins at the surface of the small intestine. Each dot represents a perfused vein. $n=5-8$ intestines. Error bars: s.e.m., ${ }^{\star \star \star} P<0.001$, Mann-Whitney U-test. Scale bars, $100 \mu \mathrm{m}$ in $\mathbf{b}-\mathbf{g}$ and $10 \mathrm{~mm}$ in $\mathbf{h}, \mathbf{j}, \mathbf{l} . \mathbf{a}$, artery in red; $\mathbf{v}$, vein in blue. 
GI tract showed staining of arterial but not venous vessels, because latex cannot perfuse capillaries (Fig. 1b,c,h,i). In contrast, both $A l k 1^{i \Delta E C_{-}}$and BMP9/10 blAB-treated mice exhibited latex dye staining of veins in retinas (Fig. 1d-g) and the GI tract (Fig. $1 \mathrm{j}-\mathrm{m}$ ). Counterstaining of retinal vessels with IsolectinB4 (IB4) showed that latex perfused AVMs with blood flowing directly from an artery into a vein without an intervening capillary bed (Fig. 1d-g). Quantification revealed an average of three latex-perfused AVMs per retina in $A l k 1^{i \Delta E C_{-}}$and two AVMs in BMP9/10 antibody-treated mice (Fig. 1n). The GI tract surface of mutants showed an average of seven in $A l k 1^{i \Delta E C}$ and four in BMP9/10 blAB perfused veins per $10 \mathrm{~mm}$ gut length, whereas no perfused veins were seen in control mice (Fig. 10).

Additional analysis of IB4-stained retinas confirmed previous results $^{15,21}$ that AVMs were usually located in the centre of the retina, close to the optic nerve, whereas increased vessel area and branching were observed at the vascular front in both $A l k 1^{i \Delta E C_{-}}$ and BMP9/10 blAB-treated mice (Supplementary Fig. 1f-k).

Sorting of endothelial cells from $A l k 1^{i \Delta E C}$ retinas showed that Alk1-deficient cells display enhanced cell cycle progression, with a significant reduction of cells in G1 phase and a concomitant increase of endothelial cells in S/G2/M (Supplementary Fig. 2a). Staining of BMP signalling-deficient retinas for smooth muscle actin (SMA) showed a decrease in arterial and an increase in venous SMA coverage when compared with control retinas, in particular in veins engaged in AVMs (Supplementary Fig. 2b-d). qPCR analysis on mLECs isolated from $A l k 1^{i \Delta E C}$ showed unchanged levels of $V e g f r 2$ but downregulation of Alk1, Eng and Vegfr 1 , as well as of Notch signalling components Notch1 and Jag1 (Supplementary Fig. 2e), in agreement with a previous report ${ }^{15}$. Arterial markers $U n c 5 b$ and $E f n b 2$ (refs 17,22) were decreased, whereas expression of the venous marker EphB4 was upregulated in Alk1 mutant cells (Supplementary Fig. 2e). Antibody staining confirmed that Alk1 mutant cells lose expression of Vegfr1 (Supplementary Fig. 2f,g), a negative regulator of VEGF signalling, and of the Notch ligand Jagged 1 (Supplementary Fig. 2h,i ${ }^{20,23}$, suggesting that reduced Notch and enhanced VEGF signalling could contribute to AVM formation in Alk1 mutant cells.

Vegfr2 deletion prevents vascular development in Alk1 mutants. To study VEGF signalling in ALK1-deficient cells, we transfected human umbilical vein endothelial cells (HUVECs) with ALK1 small interfering RNA (siRNA), which decreased ALK1 messenger RNA levels by $>90 \%$ (Fig. 2a). Compared with $\mathrm{Ctrl}$ siRNA, ALK1-depleted cells displayed equal levels of total VEGFR2 but showed an increase in VEGFR2 phosphorylation and activation of downstream pERK and pAKT in response to 10 ng VEGFA (Fig. 2b,c). Of note, pAKT was already increased at baseline in ALK1 knockdown cells (Fig. 2b,c) in the absence of exogenous VEGF.

We reasoned that blocking the Vegf signal-transducing receptor Vegfr2 (ref. 20) might prevent vascular malformations in Alk1 mutant retinal vessels. To block Vegfr2 genetically, we used $V e g f r 2^{\mathrm{fl} / \mathrm{fl}}$ mice ${ }^{24}$ intercrossed with $A l k 1^{i \Delta E C}$ mice. One hundred micrograms of Tx was administered at P3 and P4, and Alk1;Vegfr $2^{i \Delta E C}$ mice were analysed at P5 (Fig. 2d). Tx-injected Cre-negative littermates were used as controls (Fig. 2d,e). Compared with control mice, combined deletion of both Vegfr2 and Alk1 produced varying degrees of vascular defects that correlated with the extent of Vegfr2 deletion (Fig. 2e-k). $A l k 1$; Vegfr $2^{i \Delta E C}$ mice had a reduced number of AVMs when compared with $A l k 1^{i \Delta E C}$ single mutants, but many retinas still exhibited AVMs (Fig. 2f,l), despite loss of Alk1 expression (Fig. 2i).
Western blot analysis of Vegfr2 expression (Fig. $2 \mathrm{j}$ ) in the lungs corresponding to the retinas in Fig. 2e-h showed that mice with inefficient Vegfr 2 deletion (33\%) could display close to normal retina vascular patterning (Fig. 2g,i). In contrast, mice with efficient Vegfr2 deletion (88\%) had strongly reduced retinal angiogenesis, in some cases displaying barely any vasculature (Fig. 2h). Quantification of 16 retinas showed that overall vascular density and branching in double mutants was significantly lower than untreated controls (Fig. 2l), indicating that Vegfr2 inhibition was dominant over Alk1 loss of function and, if complete, prevented retinal vascular development.

Increased PI3K signalling in Alk1-deficient endothelial cells. As ALK1 knockdown HUVECs exhibited increased AKT phosphorylation in the absence of VEGF stimulation (Fig. 2b,c), we investigated activation of the PI3K/AKT pathway in HUVECs (Fig. 3a,b) and mLECs (Fig. 3c,d) treated with BMP9. Two hours after treatment, AKT phosphorylation at serine 473 and phosphorylation of downstream FOXO1 were both strongly decreased compared with untreated cells (Fig. 3a-d). Upstream of PI3K-AKT, we found that phosphorylation of the Ser380/Thr382/ Thr383-inactive form of the lipid phosphatase and tensin homologue $(\mathrm{PTEN})^{25,26}$ was decreased in BMP9-stimulated HUVECs and mLECs (Fig. 3a-d), suggesting that AKT activity might be repressed by stabilizing PTEN at the cell membrane. Total PTEN levels were also decreased by BMP9 treatment (Fig. 3a,c). We next confirmed that BMP9/ALK1 signalling regulates AKT activation by using ALK1-depleted HUVECs and mLECs or cells treated with anti-BMP9/10 blABs (Fig. 3e-h). Cells were cultured in medium complemented with $2 \%$ fetal calf serum containing BMP9 proteins ${ }^{21}$. ALK1-depleted cells and BMP ligand blockade both increased pAKT, pFOXO1 and pPTEN levels (Fig. 3e-h).

In line with the western blotting results, immunostaining of $A l k 1^{i \Delta E C}$ mLECs showed an increase in PI3K-Akt signalling. Compared with control cells, pFoxol was increased in $A l k 1^{i \Delta E C}$ cells (Fig. 3i,j), whereas nuclear Foxol staining, which is inhibited by phosphorylation ${ }^{27}$, was decreased (Fig. 3k,1). pPten immunostaining in Ctrl mLECs revealed both nuclear and membrane staining, whereas we observed an increase in nuclear and a decrease in membrane staining in $A l k 1^{i \Delta E C}$ cells (Fig. $3 \mathrm{~m}, \mathrm{n}$ ), suggesting that BMP inhibits PI3K-Akt activity via regulation of Pten localization and activity ${ }^{25,26}$.

PI3K inhibition improves AVMs in Alk1 mutants. The data suggested that inhibition of $\mathrm{PI} 3 \mathrm{~K}$ signalling might improve vascular malformations in $A l k 1^{i \Delta E C}$ mice. To test this hypothesis, we injected $50 \mu \mathrm{g}$ Tx into P3 $A l k 1^{i \Delta E C}$ mice and administered Wortmannin (PI3Ki, $0.4 \mathrm{mg} \mathrm{kg}^{-1}$, i.p.) at P3, P4 and P5 (Fig. 4a). Tx-injected Cre-negative littermates were used as controls and retinas were analysed at P5. Western blotting on whole lung lysates showed that PI3Ki efficiently inhibited AKT phosphorylation, without affecting total AKT levels (Fig. 4b). PI3Ki induced a decrease of retinal vasculature density and the number of branch points when compared with untreated Ctrl mice (Fig. 4c,d). PI3Ki also efficiently normalized the vasculature of $A l k 1$ mutants (Fig. $4 \mathrm{f}$ compared with Fig. 4e). Quantification showed that PI3Ki treatment prevented AV shunt formation in $A l k 1^{i \Delta E C}$ retinas and normalized vessel density, as well as the number of branch points close to control levels (Fig. 4g). We also found that PI3K inhibition in $A l k 1^{i \Delta E C}$ normalized the increased proliferation of the endothelial cells, which was assessed by $\mathrm{PH} 3$ staining (Fig. 4h).

We next tested the efficiency of PI3Ki in rescuing AVM formation in the internal organs. In latex-perfused control versus $A l k 1^{i \Delta E C}$ mice, PI3Ki efficiently reduced the number of 
a

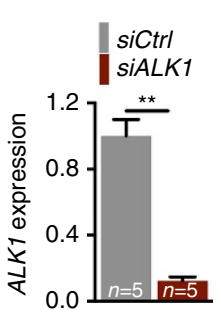

b

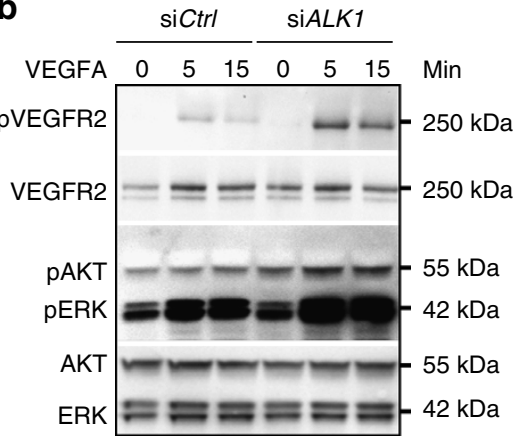

C

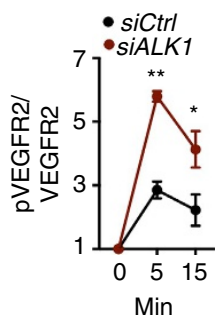

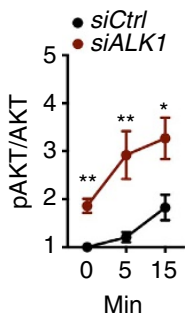

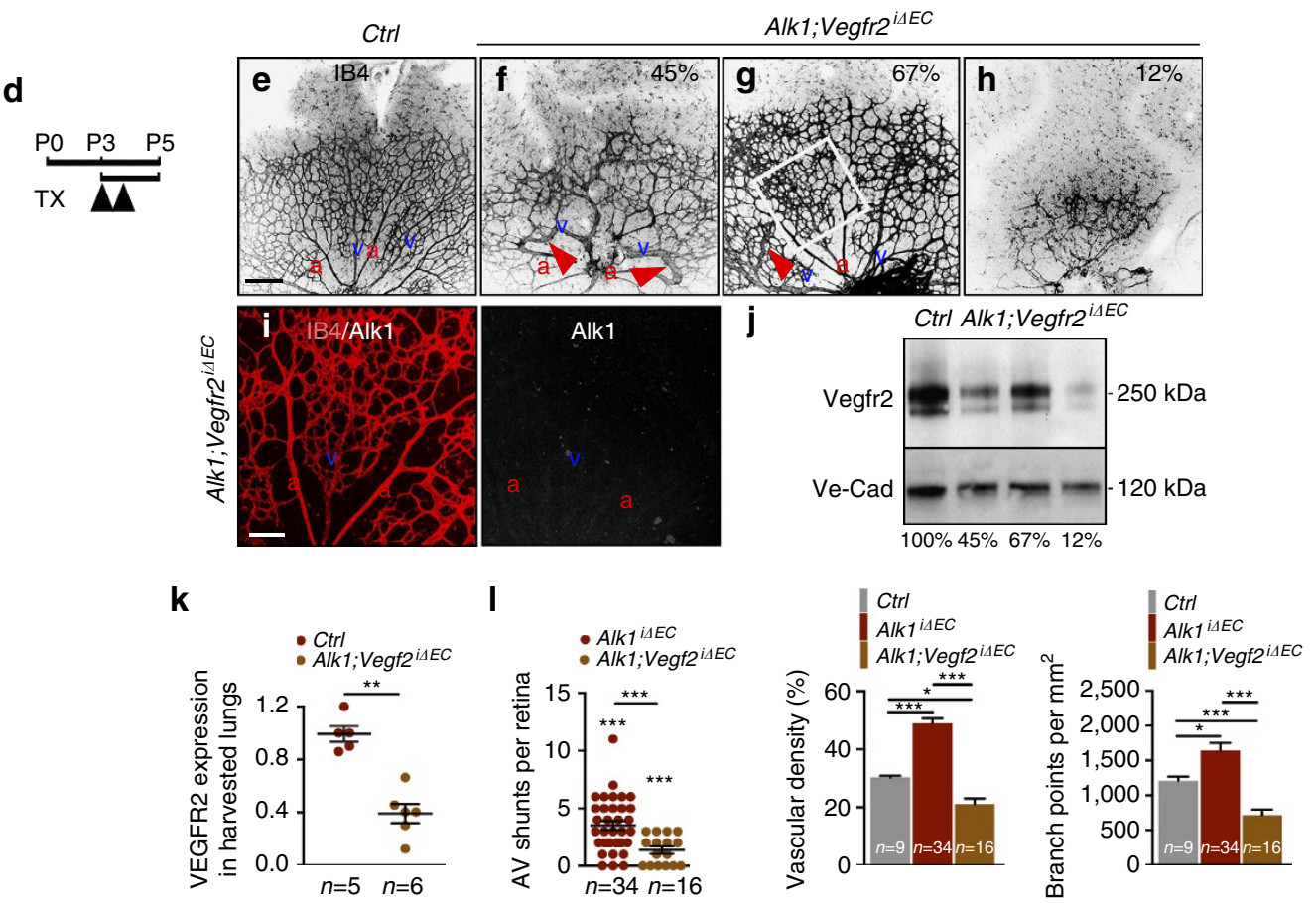

Figure 2 | Vegfr2 deletion blocks angiogenesis in Alk $\boldsymbol{1}^{\mathbf{i} A E C}$ mice. (a) ALK1 expression analysis by qPCR in HUVECs transfected with Ctrl or ALK1 siRNA. (b) Western blot analysis for VEGFR2, AKT and ERK activation in HUVECs transfected with Ctrl or ALK1 siRNA and treated with 10 ng VEGFA for 0-15 min. (c) Quantification of western blottings corresponding to $\mathbf{b}$ ( $n=5$ independent experiments). Error bars: s.e.m., ${ }^{\star} P<0.05$ and ${ }^{\star \star} P<0.01$, Mann-Whitney $U$-test. (d) Schematic representation of the experimental strategy to assess vasculature in Alk1; Vegfr2 ${ }^{i \Delta E C}$ P5 mice. Arrowheads indicate intra-gastric injections of $100 \mu \mathrm{g} \mathrm{Tx}$ at P3 and P4. (e-h) IB4 staining of retinal flat mounts (negative images of the fluorescent signal) from Ctrl mice (e) and three different Alk1;Vegfr2 ${ }^{i \Delta E C}$ P5 mice (f-h). Red arrowheads in $\mathbf{f}, \mathbf{g}$ indicate AV shunts. (i) IB4 and Alk1 double labelling of the Alk1;Vegfr2 ${ }^{i \Delta E C}$ retina shown in $\mathbf{g}$ (white square). (j) Vegfr2 protein expression in P5 lung lysates harvested from the mice shown in $\mathbf{e}-\mathbf{h}$. Percentage of residual Vegfr2 expression in each lysate is indicated in the corresponding panels in $\mathbf{f}-\mathbf{h}$ and below the blot. (k) Quantification of Vegfr2 protein normalized to Ve-Cadherin detected by western blotting shown in j. Each dot represents 1 lung. $n=5-6$ mice per group. Error bars: s.e.m., ${ }^{\star \star} P<0.01$, Mann-Whitney U-test. (I) Quantification of AV shunt number, vascular density and number of branch points. Each dot represents one retina. $n=9-34$ Retinas per group. Error bars: s.e.m., ${ }^{\star} P<0.05$ and ${ }^{\star \star \star} P<0.001$, Mann-Whitney $U$-test. Scale bars, $200 \mu \mathrm{m}$ in $\mathbf{e}-\mathbf{h}$ and $100 \mu \mathrm{m}$ in $\mathbf{i}$ a, artery in red; $v$, vein in blue.

latex-perfused retinal AVMs and veins on the surface of the GI tracts (Fig. 4i-o).

To confirm the effects of PI3K pathway inhibition in rescuing AVMs in $A l k 1^{i \Delta E C}$ mice we used another PI3K inhibitor, Pictilisib (PI3Ki 2) ${ }^{28}$. Alk1 deletion was induced at P3 with $50 \mu \mathrm{g}$ Tx and $20 \mathrm{mg} \mathrm{kg}^{-1}$ of PI3Ki2 was administered i.p. at P3-P5 and retinal vasculature was analysed at P5 (Supplementary Fig. 3a). Staining for IB4 shows that inhibition of PI3K activity with PI3Ki2 efficiently prevented the development of AV shunts and rescued vascular defects in $A l k l^{i \Delta E C}$ retinas (Supplementary Fig. 3b-d).

PI3Ki treatment also prevented vascular defects induced by BMP9/10 blockade (Fig. 5a-d). BMP9/10 blAB and PI3Ki were administered at P3 and P4, and P3-P5, respectively, and the retinal vasculature was analysed at P5 (Fig. 5a). Staining for IB4 showed that inhibition of PI3K activity efficiently prevented the development of AV shunts and rescued vascular defects induced by BMP9/10 blAB (Fig. 5b-d).

To confirm the efficacy of PI3K inhibition in vivo, treated versus untreated controls, versus $A l k 1^{i \Delta E C}$ and BMP9/10 blAB-injected mice retinas were labelled for PI3K downstream signalling components (Fig. 6a-m). Endothelial nuclear Foxo1 staining ${ }^{29}$ was strongly decreased in Alk1 mutant and BMP9/10 blAB-treated mice (Supplementary Fig. 4a-d). Staining with phospho-S6 (ref. 30), which is positively regulated by PI3K-AKT signalling was strongly increased in BMP9/10 blAB- (Fig. 6b,c) and $A l k 1^{i \Delta E C}$-treated retinas (Fig. 6e,h) when compared with 
a

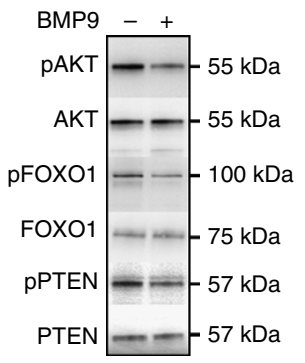

e

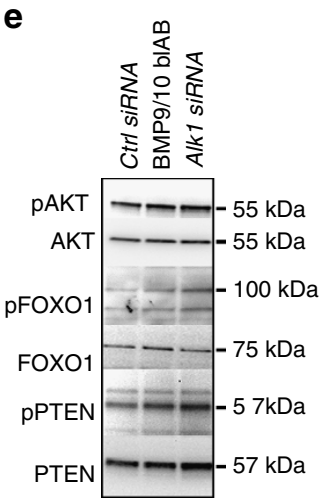

b

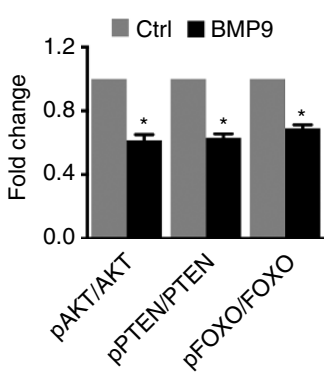

f

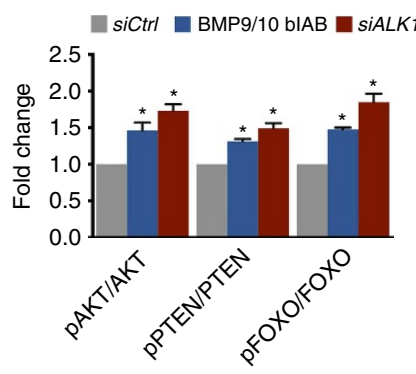

C

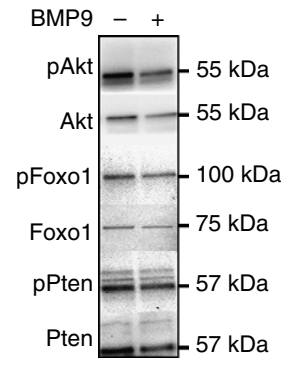

g

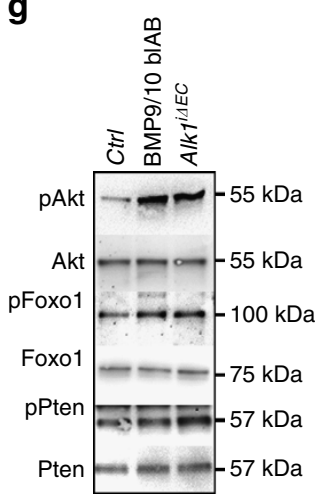

d

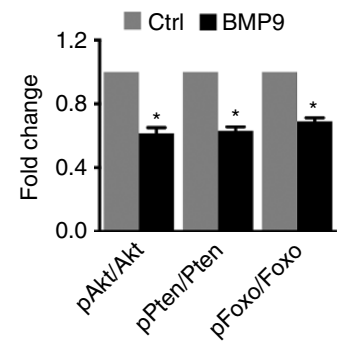

h

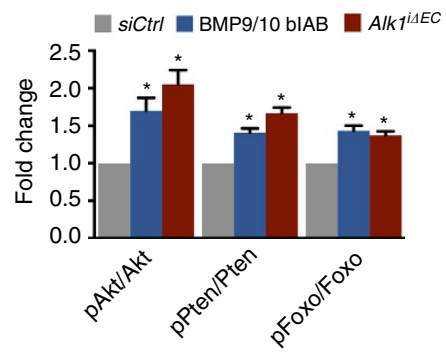

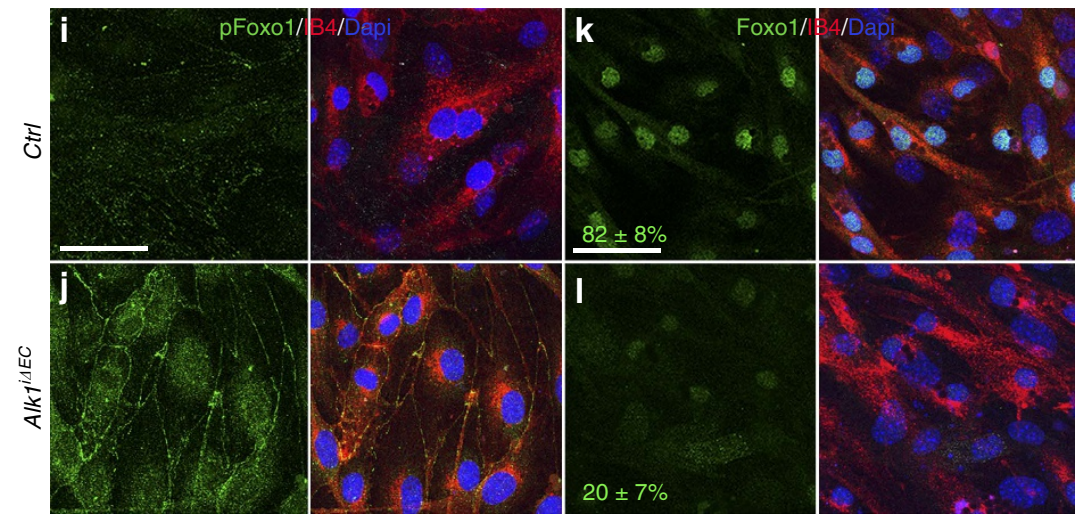
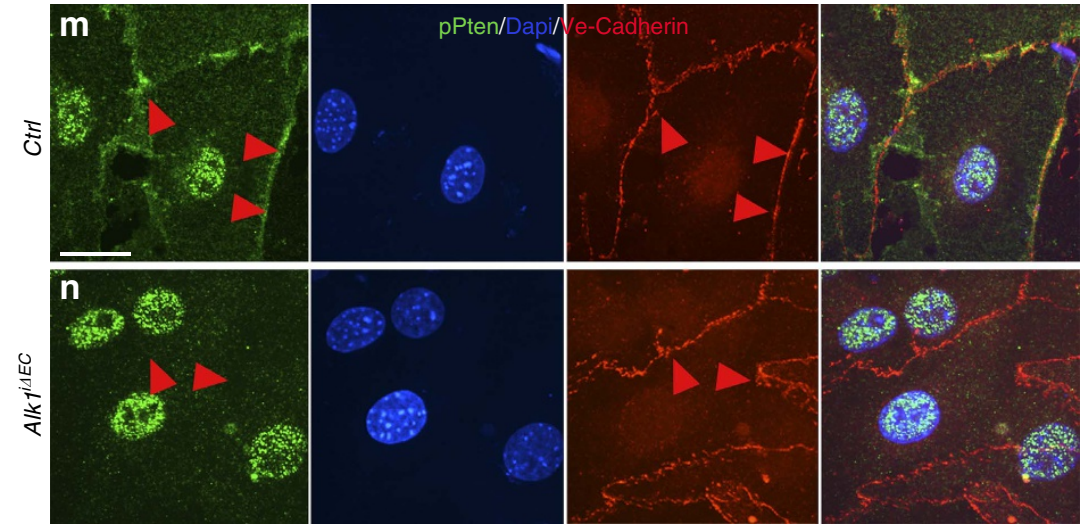

Figure 3 | Alk1 deletion increases PI3K signalling. (a,c) Western blot $(\mathrm{Wb})$ analysis of HUVECs $(\mathbf{a})$ and $\mathrm{mLECs}(\mathbf{c})$ stimulated or not with BMP9 for $2 \mathrm{~h}$. $(\mathbf{b}, \mathbf{d})$ Quantifications of HUVECs (b) and mLECs (d) pAKT, pPTEN and pFOXO1 levels normalized to total AKT, PTEN and FOXO1, respectively. (e) Wb analysis of HUVECs transfected with Ctrl or ALK1 siRNA or treated with $1 \mu \mathrm{g} \mathrm{ml} \mathrm{m}^{-1}$ of BMP9/10 blAB for $36 \mathrm{~h}$. (f) Quantifications of pAKT, pPTEN and pFOXO1 levels normalized to total AKT, PTEN and FOXO1, respectively. (g) Wb analysis of $\mathrm{mLECs}$ with the indicated genotype or treated with $1 \mu \mathrm{g}$ ml ${ }^{-1}$ of BMP9/10 blAB for 36 h. (h) Quantifications of pAkt, pFoxo1 and pPten levels normalized to total Akt, Foxo1 and Pten, respectively. Graphs represent mean of $n=4$ experiments. Error bars: s.e.m., ${ }^{\star} P<0.05$, Student's T-test. (i,j), pFoxo1 (green), IB4 (red) and Dapi (blue) staining of Ctrl (i) and Alk ${ }^{i}{ }^{14 E C}$ (j) $\mathrm{mLECs}$ in culture. (k,I) Foxo1 (green), IB4 (red) and Dapi (blue) staining for Ctrl (k) or Alk $7^{\text {iAEC }}$ (I) mLECs. Numbers represent percentage of cells with nuclear Foxo1 staining. (m,n) Anti-pPten (green), Dapi (blue) and Ve-Cadherin (red) staining for Ctrl (m) and Alk $7^{i \Delta E C}$ (n) mLECs in culture. Red arrowheads indicate pPten expression at plasma membrane. Scale bars, $100 \mu \mathrm{m}$ in $\mathbf{i}-\mathbf{I}$ and $10 \mu \mathrm{m}$ in $\mathbf{m}, \mathbf{n}$. 
a

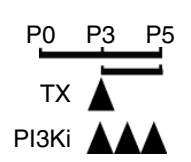

b

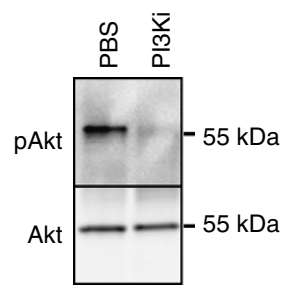

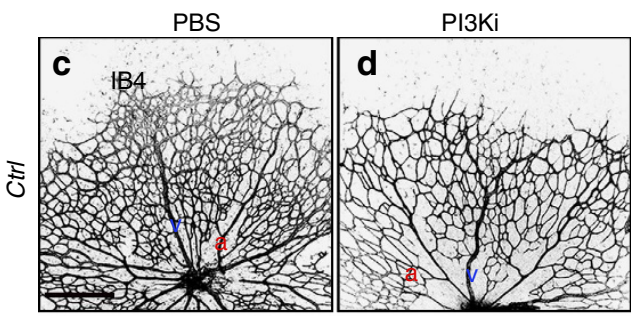

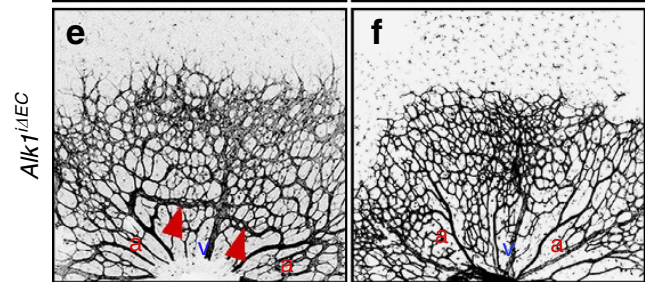

g

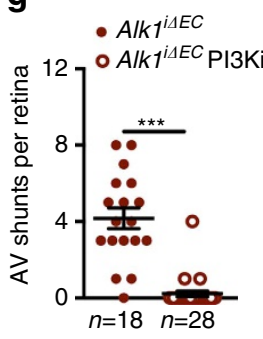

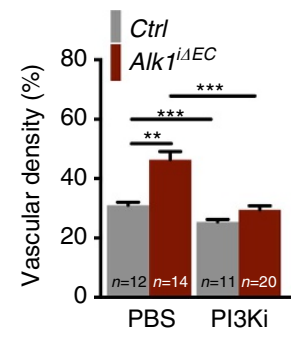

$A l k 1^{i \Delta E C}$

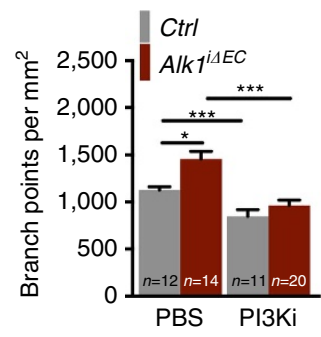

h
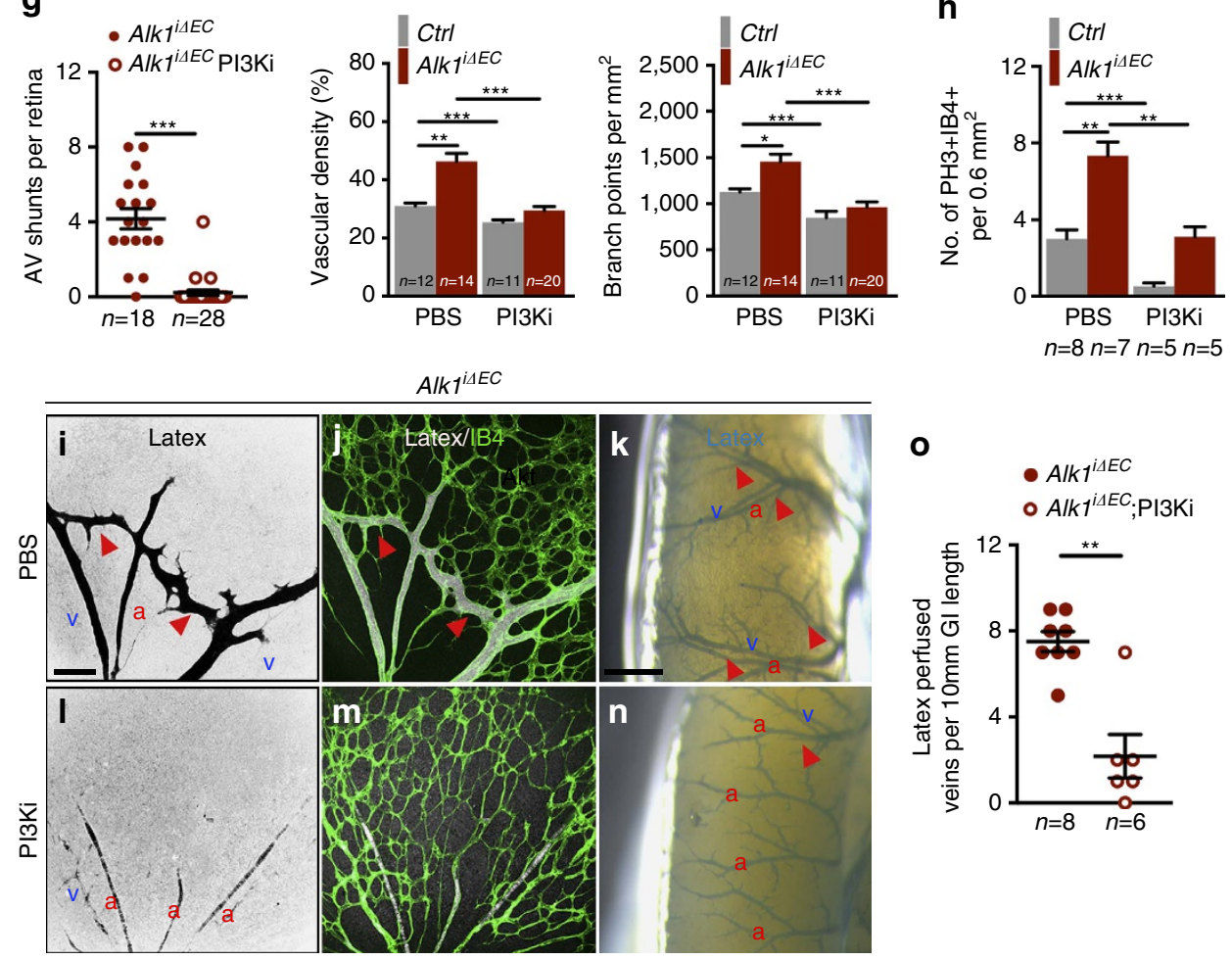

o

$$
\begin{aligned}
& \text { - } A / K 1^{i \Delta E C} \\
& \text { - } A l k 1^{14 E C} ; \mathrm{PI} I 3 \mathrm{Ki}
\end{aligned}
$$

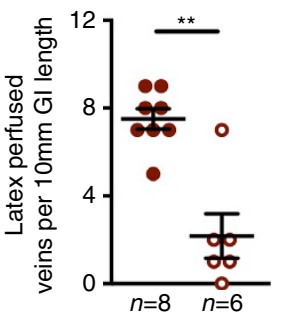

Figure 4 | PI3K inhibition prevents vascular defects in Alki ${ }^{\text {iAEC }}$ mice. (a) Schematic representation of the experimental strategy to assess the effects of PI3K inhibition on Alk $7^{i \Delta E C}$ vasculature. Arrowheads indicate the time course of Tx ( $50 \mu \mathrm{g}$ ) and Wortmannin (PI3Ki, $0.4 \mathrm{mg} \mathrm{kg}^{-1}$ ) or PBS (vehicle) administration. (b) Western blot analysis of Akt activation (pAkt) in total lung lysates from PBS or PI3K inhibitor-treated wild-type mice. (c-f) IB4 staining of retinal flat mounts (negative images of the fluorescent signal) from $\mathrm{Ctrl}(\mathbf{c}, \mathbf{d})$ and $A l k 7^{i \Delta E C}$ P5 mice $(\mathbf{e}, \mathbf{f})$ injected with vehicle $(\mathbf{c}, \mathbf{e})$ or PI3Ki (d,f). Red arrowheads indicate AV shunts. (g) Quantification of the AV shunt number, vascular density and number of branchpoints. Each dot represents one retina. $n=11-28$ retinas per group. Error bars: s.e.m., ${ }^{\star} P<0.05$, ${ }^{\star \star} P<0.01$ and ${ }^{\star \star \star} P<0.001$, Mann-Whitney U-test. (h) Quantification of PH3 +, IB4 + endothelial cells of P5 retinas harvested from mice with the indicated genotypes treated with PBS or PI3Ki. $n=5-8$ retinas per group. Error bars: s.e.m., ${ }^{\star \star} P<0.01$ and ${ }^{\star \star \star} P<0.001$, Mann-Whitney U-test. (i,j, I, m) Vascular staining with latex dye (black in single images in $\mathbf{i}, \mathbf{I}$ and white in merged images in $\left.\mathbf{j}, \mathbf{m}\right)$ and IB4 (green) of retinal flat mounts from Alk $7^{i \Delta E C}$ P5 mice treated with PBS $(\mathbf{i}, \mathbf{j})$ or PI3Ki $(\mathbf{I}, \mathbf{m})$. ( $\left.\mathbf{k}, \mathbf{n}\right)$ Vascular staining of the small intestine surface (blue) of Alk $7^{i \Delta E C}$ treated with PBS (k) or PI3Ki (n) P5 mice. Red arrowheads in i-k,n indicate latex-perfused veins. (o) Quantification of latex-perfused veins. Each dot represents one intestine. $n=6-8$ Intestines per group. Error bars: s.e.m., ${ }^{\star \star} P<0.01$, Mann-Whitney U-test. Scale bars, $200 \mu \mathrm{m}$ in $\mathbf{c}-\mathbf{f}, 100 \mu \mathrm{m}$ in $\mathbf{i}, \mathbf{l}$ and $500 \mu \mathrm{m}$ in $\mathbf{k}, \mathbf{n}$. a, artery in red; $v$, vein in blue.

control retinas (Fig. 6a,d). PI3Ki treatment decreased the expression of phospho-S6 in controls (Fig. 6f,h) and in Alk1 mutant retinas (Fig. 6g,h). Furthermore, Eng, which is induced by Alk1 signalling ${ }^{15}$ and repressed by PI3K (ref. 31), was decreased in Alk1 mutant retinal vessels (Fig. 6j,m) when compared with control retinas (Fig. 6i,m) and the expression was rescued by PI3Ki treatment (Fig. 61,m).
PI3K inhibition reverts established AVMs in Alk1 mutants. To address whether inhibition of PI3K was able to revert already established AVMs, we first determined the time course of AVM

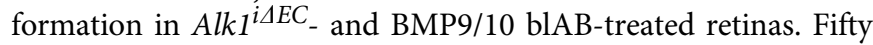
micrograms of Tx or BMP9/10 blABs were administered at P3 in $A l k 1^{i} S E C$ or control pups, respectively, and retinas were analysed 24 (P4) or $40 \mathrm{~h}$ later (P5) (Fig. 7a). Seventy per cent of $A l k 1^{i \Delta E C}$ 

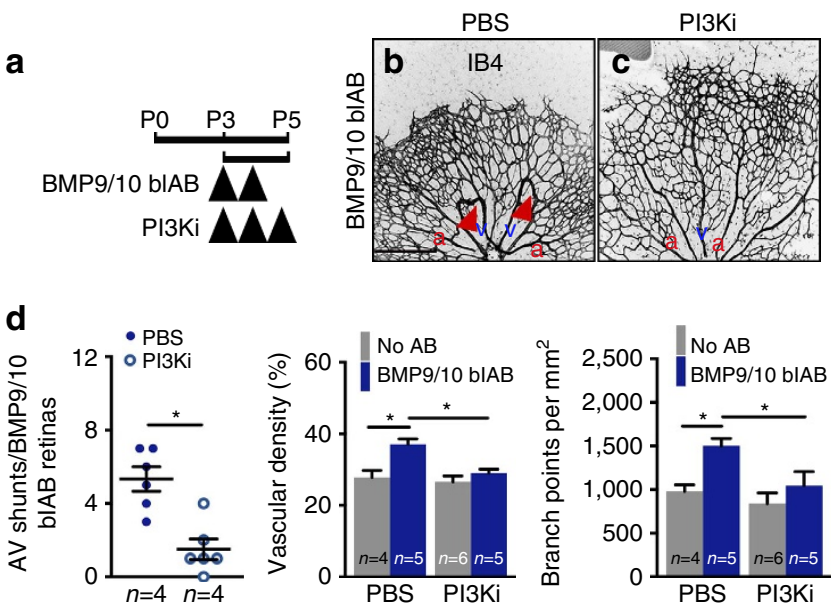

Figure 5 | PI3K inhibition prevents AVM formation in BMP9/10 bIAB-

treated mice. (a) Schematic representation of the experimental strategy to assess the effects of PI3K inhibition on the retinal vasculature of BMP9/10 bIAB injected mice. Arrowheads indicate the time course of injection of $\mathrm{BMP} 9 / 10$ bIAB and PI3Ki or PBS. $(\mathbf{b}, \mathbf{c})$ IB4 staining of retinal flat mounts from mice treated with BMP9/10 bIAB (b) and BMP9/10 bIAB and PI3Ki (c). Red arrowheads in $\mathbf{b}$ indicate AV shunts. Scale bars, $200 \mu \mathrm{m}$ in b,c. (d) Quantification of AV shunt number, vascular density and number of branch points. $n=4-6$ Retinas per group. Each dot represents one retina. Error bars: s.e.m., ${ }^{\star} P<0.05$, Mann-Whitney U-test. a, artery in red; $v$, vein in blue.

retinas and $87 \%$ of $\mathrm{BMP} 9 / 10 \mathrm{blAB}$-treated retinas exhibited at least one AV shunt $24 \mathrm{~h}$ after injection (Fig. 7b). Staining for the endothelial nuclear Erg1,2,3 transcription factor ${ }^{32}$ and Ve-Cadherin showed an increase in the number of endothelial cells specifically at branches of retinal veins (Fig. 7c-g). In control retinas, these branches contained a single cell (Fig. 7c), whereas $A l k 1^{i \Delta E C}$ or BMP9/10 blAB-treated mice showed two to three cells (Fig. $7 \mathrm{~d}-\mathrm{f}$ ). At $40 \mathrm{~h}$, both arterial and venous branches in mutant AVMs exhibited more than three cells compared with one cell in controls (Fig. 7c-g), suggesting that AVMs arise from the enlargement of capillaries and an increase in the cell number at venous and arterial branch points.

Having established that AVMs were present at P4, we next induced gene deletion with $50 \mu \mathrm{g} \mathrm{Tx}$ at P3 and administered PI3Ki 24 and $40 \mathrm{~h}$ later. Pups were killed at P5 (Fig. 7h). Analysis of retinal vasculature showed that PI3Ki efficiently rescued vascular anomalies in $A l k 1^{i \triangle E C}$ retinas (Fig. $7 \mathrm{i}-\mathrm{k}$ ). Likewise, PI3Ki treatment at P4 after induction of BMP9/10 blockade at P3 and P4 also reduced vascular defects (Fig. 7l-o), suggesting that inhibition of $\mathrm{PI} 3 \mathrm{~K}$ pathway reverts already established AVMs.

\section{Discussion}

The major novel finding reported here is that inhibition of BMP9 and 10 signalling through the Alk1 receptor leads to overactivation of the PI3K-Akt pathway and that inhibition of PI3K activity rescues vascular malformations in mouse models of HHT2 (Fig. 8). The PI3K/Akt pathway stimulates endothelial cell proliferation, migration and survival downstream of various angiogenic growth factors ${ }^{33}$. We show that pharmacological inhibition of PI3K signalling using two different inhibitors prevents and even rescues abnormal features in BMP signallingdeficient retinas and the GI tract. Inhibition of PI3K in wild-type retinas closely mimics the retinal angiogenesis phenotype in mice lacking the $P I 3 K 110 \alpha$ isoform or Akt1 gene ${ }^{34,35}$ confirming treatment specificity. However, PI3K inhibition rescued excessive retinal endothelial cell proliferation, providing one possible mechanism for its effects in vivo. In addition, a recent study showed that AVM formation in zebrafish Alk1 mutants was due to altered endothelial cell migration ${ }^{36}$, which in mouse retinas is dependent on the PI3K110 $\alpha$ isoform ${ }^{34}$. Therefore, with increased endothelial cell proliferation, altered migration is likely to be implicated in the Alk1 mutant phenotype and may be targeted by PI3K inhibition. This pathway therefore represents an attractive potential therapy for Alk1-mediated HHT2 disease.

Mechanistically, we found that enhanced PI3K-Akt pathway activation following BMP9 signalling blockade involves the upstream lipid phosphatase Pten (Fig. 8). BMP9 was previously shown to decrease Pten expression levels in mouse embryonic fibroblasts ${ }^{37}$, which prompted us to investigate Pten as a possible upstream regulator of PI3K-Akt phosphorylation. We confirmed changes in total Pten levels and additionally found more dramatic effects on the C-terminal Pten phosphorylation. Pten catalyses the conversion of phosphatidylinositol 3,4,5-trisphosphate into phosphatidylinositol 4,5-bisphosphate, thereby counteracting PI3K-Akt signalling at the cell membrane. When phosphorylated, Pten assumes a closed conformation with an inactive phosphatase domain that is unable to bind the cell membrane and to dephosphorylate PI3K substrate, thereby increasing PI3K activity ${ }^{25,38}$.

Our in vitro experiments show that BMP9 treatment decreases carboxy-terminal PTEN phosphorylation in HUVECs and mLECs, thereby increasing PTEN activity at the cell membrane, leading to reduced phosphorylation of AKT and of downstream FOXO1 transcription factor. Conversely, loss-of-function experiments using Alk1 knockout mLECs or knockdown HUVECs, or BMP9/10 blAB-treated mLECs and HUVECs show the reverse effect: increased PTEN phosphorylation led to increased AKT and FOXO1 phosphorylation. This points to regulation of C-terminal PTEN phosphorylation by BMP9 as the critical step for AKT activation (Fig. 8). We are currently investigating the mechanistic basis for this effect. BMP9/ALK1 might signal via SMAD proteins to repress transcription of PTEN and/or induce kinases targeting the PTEN C terminus.

PTEN mutations in humans cause vascular anomalies ${ }^{39}$, suggesting PTEN as a major hub to maintain the proper balance between BMP and PI3K signalling pathways. Likewise, overactivation of the $\mathrm{PI} 3 \mathrm{~K} / \mathrm{AKT} /$ mammalian target of rapamycin pathway has been shown to cause vascular anomalies. Activating mutations of $A K T /$ Protein kinase B (ref. 40), TIE2 receptor (ref. 41) or PIK3CA (ref. 42) have been associated with the development of vascular anomalies in mice and humans. Interestingly, the absence of AV shunts in these pathologies, or in Pten-deficient mice retinas suggests that an increase in PI3K signalling by itself is not sufficient to trigger $\mathrm{AVMs}^{28}$. One could speculate that increased PI3K signalling leads to AVMs only in the context of reduced Smad or Notch signalling. Regardless of the precise mechanism, the data shown here demonstrate that blocking BMP9/10 signalling induces an increase in PI3K signalling and identify PI3K signalling pathway inhibition as a putative novel therapeutic approach for HHT2 patients.

PI3K signalling is activated by various growth factors, including $\mathrm{VEGF}^{43}$. Our data show that VEGF signalling is increased in ALK1 mutant cells. Several possible causes for enhanced VEGF signalling exist: first, hypersprouting and AVM formation in BMP9/10-Alk1 signalling-deficient retinas probably leads to hypoxia, which is known to increase Vegf levels, and VEGF levels are also increased in human HHT patients ${ }^{44}$. Second, we and others have previously shown that BMP9 treatment leads to an increase in the expression of Notch signalling components Hey1 and Hey2, as well as in Vegfr1, and Notch and Vegfr1 counteract Vegf signalling through Vegfr2 in 

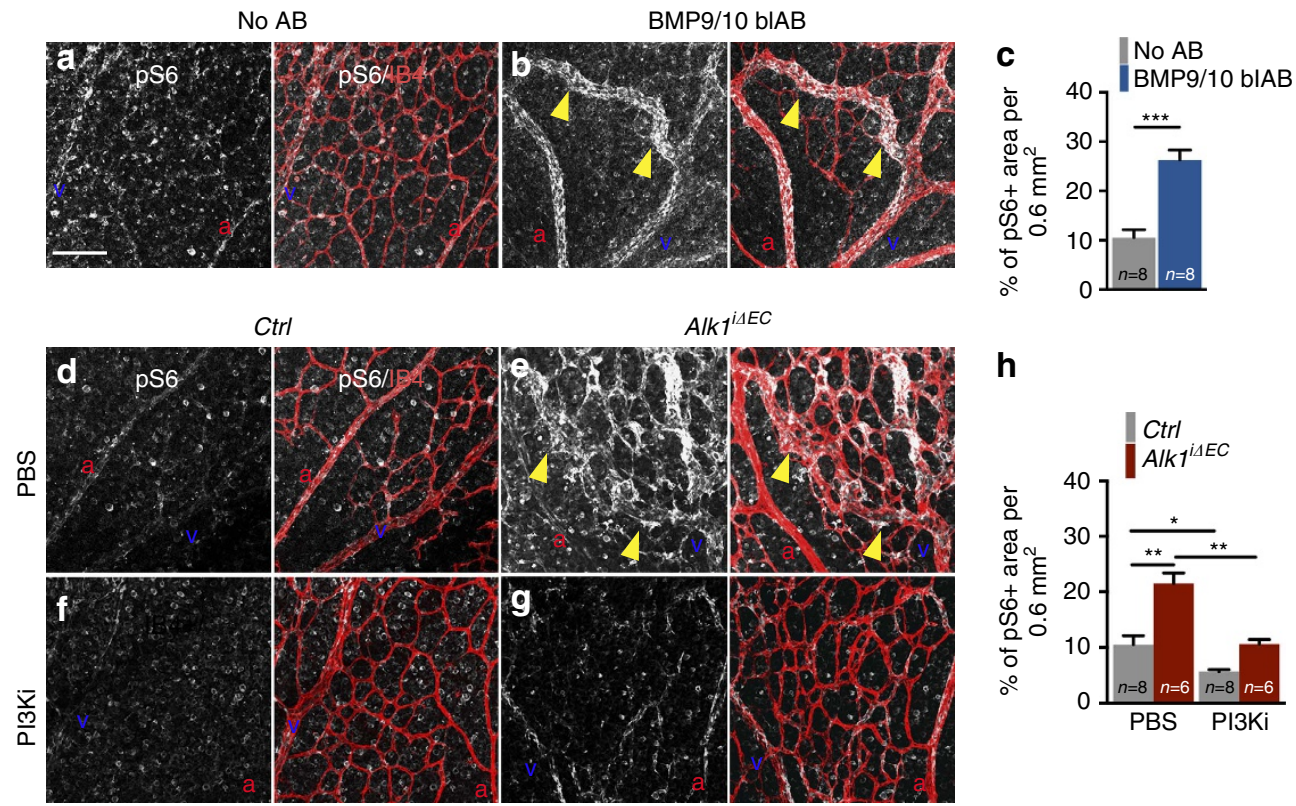

$\mathbf{h}$
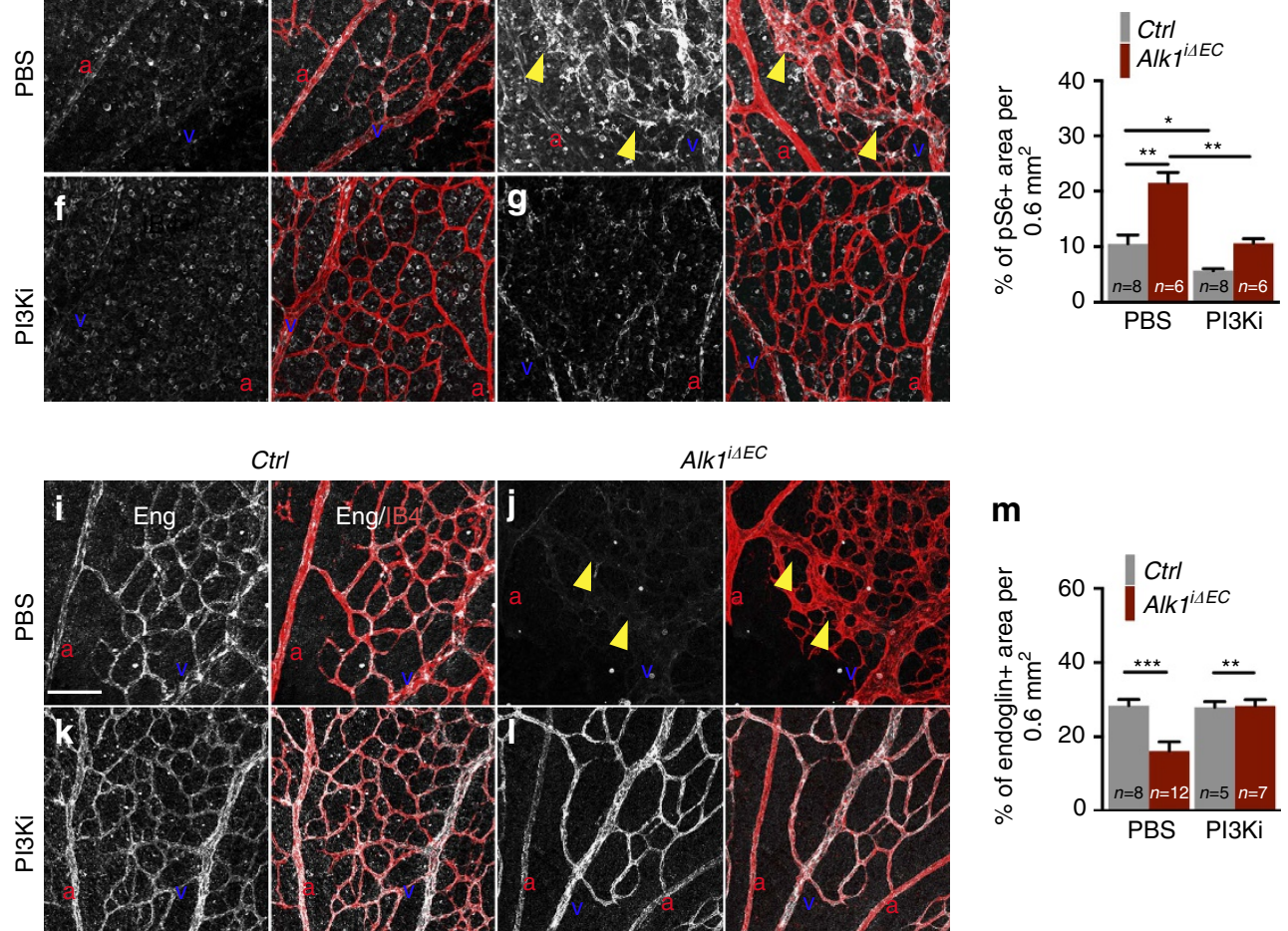

m

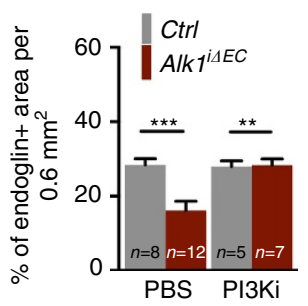

Figure 6 | PI3K inhibition rescues downstream signalling components. (a,b) Anti phospho-S6 (pS6) and IB4 staining of retinal flat mounts from P5 mice treated or not with BMP9/10 bIAB. (c) Quantification of the percentage of pS6 + vascular area per field of view. $n=8$ Retinas per group. Error bars: s.e.m., ${ }^{\star \star \star} P<0.001$, Mann-Whitney U-test. (d-g) Anti-pS6 and IB4 staining of retinal flat mounts from Ctrl (d,f) or Alk7 ${ }^{i \Delta E C}(\mathbf{e}, \mathbf{g})$ treated with PBS $(\mathbf{d}, \mathbf{e})$ or PI3Ki $(\mathbf{f}, \mathbf{g})$. (h) Quantification of the percentage of pS6+ vascular area per field of view. $n=6-8$ Retinas per group. Error bars: s.e.m., ${ }^{\star} P<0.05$ and ${ }^{\star \star} P<0.01$, Mann-Whitney U-test. (i,I) Anti-Eng and IB4 staining of retinal flat mounts from Ctrl $(\mathbf{i}, \mathbf{k})$ or Alk $7^{i \Delta E C}(\mathbf{j}, \mathbf{I})$ treated with PBS $(\mathbf{i}, \mathbf{j})$ or PI3Ki $(\mathbf{k}, \mathbf{I})$. ( $\left.\mathbf{m}\right)$ Quantification of the percentage of Eng + vascular area per field of view. Yellow arrowheads in $\mathbf{b}, \mathbf{e}, \mathbf{j}$ indicate AV shunts. $n=5-12$ Retinas per group. Error bars: s.e.m., ${ }^{\star \star} P<0.01$ and ${ }^{\star \star \star} P<0.001$, Mann-Whitney $U$-test. Scale bars, $100 \mu \mathrm{m}$ in $\mathbf{a}, \mathbf{b}, \mathbf{d}-\mathbf{g}, \mathbf{i}-\mathbf{I}$. a, artery in red; $v$, vein in blue.

retina vessels ${ }^{15,45-47}$. Chromatin immunoprecipitation sequencing analysis had shown that Smad1,5 binding to the promoter of Hey1 and Hey2 was increased after BMP9 treatment of HUVECs $^{48}$, suggesting that BMP9-Smad directly activates Notch signalling components. We and others ${ }^{15}$ also observe that $A l k 1$ mutant endothelial cells show a corresponding decrease in Vegfr1 and Notch signalling components. Although we did not see effects on Vegfr2 levels, Hey2 (HESR1) was previously shown to decrease VEGFR2 promoter-luciferase reporter activity ${ }^{49}$. Decreased VEGFR1 levels may be sufficient to account for the enhanced VEGF signalling shown in Fig. 2b; alternatively, VEGFR2 levels may change transiently at different time points.

We tested whether blocking excessive VEGF could rescue vascular defects in Alk1 mutants. Administration of Vegf blAB in temporally inducible Alk1 mouse mutants has been shown to block progression of cutaneous $\mathrm{AV}$ shunts ${ }^{50}$. Furthermore, VEGF-blAB is currently tested in clinical trials in HHT patients $^{51-53}$. We show that deletion of Vegfr2, the main signal transducing receptor for Vegf, prevents angiogenesis, but does not fully rescue normal vascular patterning and AVM formation.
In fact, the severity of the vascular phenotype in Alk1;Vegfri $2^{i \Delta E C}$ mice was Vegfr2 dose dependent. We found that Cdh5-CreERT2mediated Vegfr2 excision was highly variable between mice, as described previously ${ }^{54}$. Proper interpretation of the results thus required correlating Vegfr2 protein levels in mouse lungs to the retinal vascular phenotype in each mouse. Using this approach, we found that strong inhibition of Vegfr2 expression severely impaired the development of retinal vasculature in Alk1 mutants, as previously shown on a wild-type background ${ }^{54}$. Vegfr 2 deletion completely abolished the hypervascular phenotype resulting from Alk1 deletion, indicating that Vegf signalling inhibition is dominant and overrides lack of Alk1 signalling. However, the retinas of the Alk1;Vegfr $2^{i \Delta E C}$ pups still displayed AV shunts, suggesting that additional signalling components may be involved in AVM formation. This idea is also supported by in vitro studies with HUVECs and mLECs, where PI3K signalling is increased in the absence of exogenous VEGF treatment. Thus, the data support the existence of a specific disease-causing enhanced PI3K activity downstream of Alk1 deficiency that is at least partially VEGF independent. Nevertheless, our data show that 
down-modulation of excessive Vegf signalling normalizes vasculature, supporting potential clinical benefits of anti-VEGF treatment in HHT2 patients.
Finally, our data confirm that BMP9/10 are physiological Alk1 ligands in mice. In previous studies, blocking of BMP10 in BMP9 mutants or ligand sequestration by Alk1-Fc treatment induced a

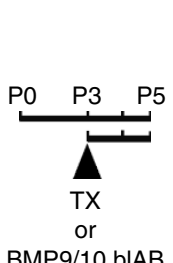

b
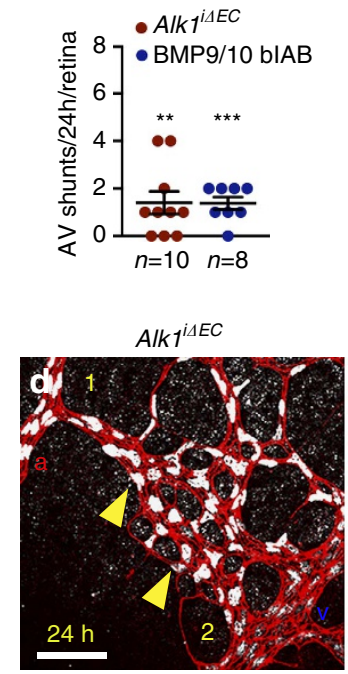

h

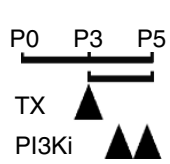

I

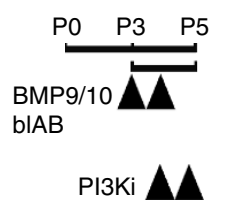

PBS

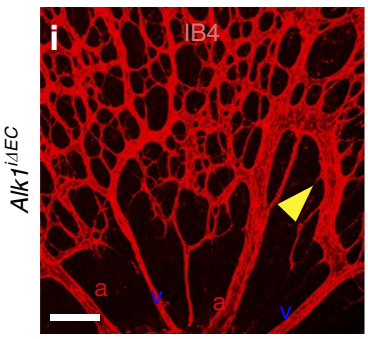

k

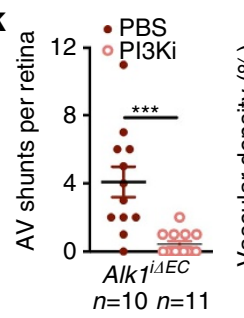

PBS
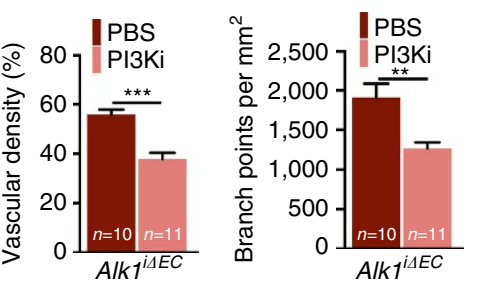

$\mathrm{PI} 3 \mathrm{Ki}$

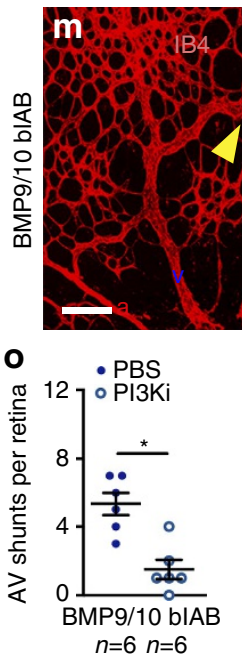

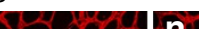
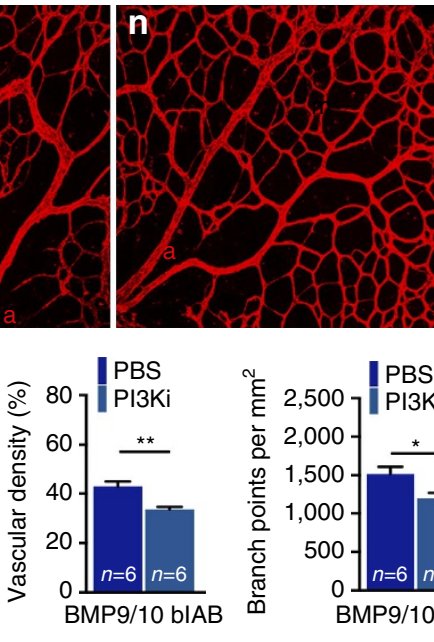

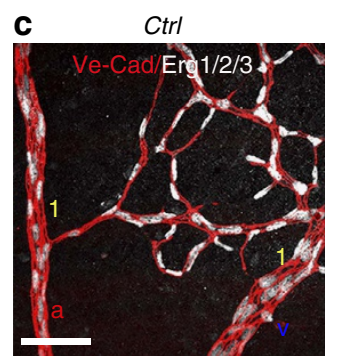

BMP9/10 bIAB

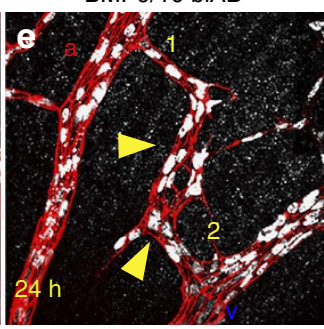

$\mathrm{PI3Ki}$

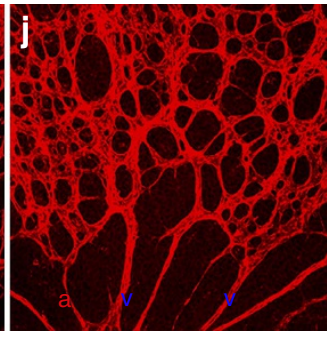

g

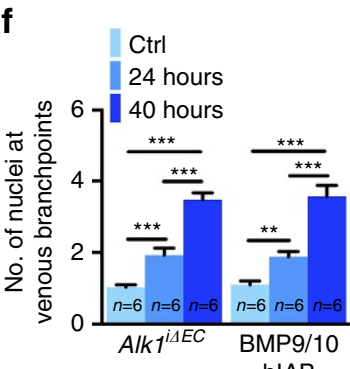

g $\quad$ Ctrl

24 hours

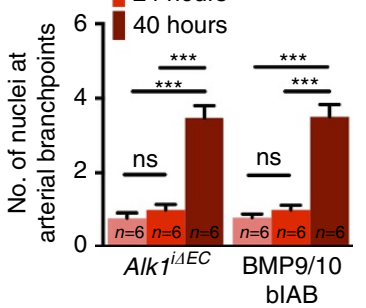


retina hypervascularization, but not AVM formation ${ }^{45,47}$, leaving open the possibility that additional Alk1 signalling mechanisms might protect from AVM formation. In these studies, treatments were administered to pups at $\mathrm{P} 1$, a stage when retina vascularization begins, and arteries and veins have not yet formed ${ }^{55}$. When receptor deletion and ligand blockade are done at P3, a stage when retina arteries and veins have formed and carry flow ${ }^{56}$, AVMs develop, suggesting that flow is required for AVM formation in mice ${ }^{21}$. Furthermore, subjecting endothelial cells to laminar shear stress potentiates signalling induced by low concentrations of BMP9 in vitro ${ }^{21}$. In zebrafish embryos, arresting flow prevents AVM formation in Alk1 mutants ${ }^{57}$, demonstrating that flow is an evolutionary conserved trigger of AVM formation. AVMs always form between the larger arteries and veins close to the optic nerve that carry most blood flow, whereas less perfused vasculature towards the angiogenic front develops hypervascularization. Thus, existing data suggest that BMP9/10 signalling through Alk1 protects vasculature from hypervascularization in low flow conditions and at the same time prevents AVM development induced by higher flow. AVMs form via enhanced cell cycle progression and enlargement of capillary connections between retinal arteries and veins. Simply put, it appears that BMP9/10 signalling through Alk1 protects capillary size at vessel branch points, thereby allowing proper branching morphogenesis of the vasculature.

\section{Methods}

Mice. All animal experiments were performed under a protocol approved by Institutional Animal Care Use Committee of Yale University.

Eight weeks old $A l k 1^{i \triangle E C}$ and $A l k 1 ; V e g f r 2^{i \triangle E C}$ mice of mixed genetic background were intercrossed for pups. Offsprings of both genders were used. Gene deletion was induced by intra-gastric injections with $50 \mu \mathrm{g}$ Tx (Sigma, T5648; $1 \mathrm{mg} \mathrm{ml}^{-1}$ ) into $A l k 1^{i \Delta E C}$ and $100 \mu \mathrm{g} \mathrm{Tx}$ into Alk1; Vegfr $2^{i \Delta E C}$ pups at P3 and P4. Tx-injected Cre-negative littermates were used as controls. BMP9/10 blABs $\left(10 \mathrm{mg} \mathrm{kg}^{-1}\right.$ per day) and the PI3K inhibitors were injected i.p.: BMP9/10 blABs between P2-P4, Wortmannin $\left(0.4 \mathrm{mg} \mathrm{kg}^{-1}\right.$ per day) or Pictilisib $\left(20 \mathrm{mg} \mathrm{kg}^{-1}\right.$ per day) once at P3, twice at P4 and once at P5.

Latex dye injection. P5 pups were anaesthetized on ice, and abdominal and thoracic cavities were opened. The right atrium was cut, blood was washed out with $2 \mathrm{ml}$ PBS and $1 \mathrm{ml}$ of latex dye (Blue latex, BR80B; Connecticut Valley Biological Supply Co.) was slowly and steadily injected into the left ventricle with an insulin syringe. Retinas and GI tracts were washed in PBS and fixed with $4 \%$ paraformaldehyde (PFA) overnight. Retinas were stained with Isolectin B4 and GI tracts were cleared in 10\% glycerol, $4 \mathrm{M}$ urea and $0.1 \%$ Triton for 1-2 weeks before imaging.

Reagents and antibodies. For immunodetection: anti-Alk1 (AF 770, $1 \mu \mathrm{g} \mathrm{ml}^{-1}$, R\&D systems), anti-Eng (AF1320, $1 \mu \mathrm{g} \mathrm{ml}^{-1}$, R\&D systems), anti-Jagged1 (AF599, $1 \mu \mathrm{g} \mathrm{ml}^{-1}, \mathrm{R} \& \mathrm{D}$ systems), anti-Vegfr1 (AF471, $2 \mu \mathrm{g} \mathrm{ml}^{-1}$, R\&D systems), anti-Erg1/2/3 (SC353, $2 \mu \mathrm{g} \mathrm{ml}^{-1}$, Santa Cruz), anti- $\alpha$-SMA CY3 (CY3-SMA, C6198, 1:200 working solution, Sigma), anti-phosphoS6 (5364, 1:200 working solution, Cell Signalling), anti-CD144 (555289, $2 \mu \mathrm{g} \mathrm{ml}^{-1}, \mathrm{BD}$ Pharmingen), IB4 (121412, $10 \mathrm{\mu g} \mathrm{ml}^{-1}$, Life Technologies), anti-pFOXO1 (9464, 1:100 working solution, Cell Signalling), anti-FOXO1 (2880, 1:100 working solution,
Cell Signalling), anti-pPTEN (9549, 1:100 working solution, Cell Signalling), anti-PTEN (9188, 1:100 working solution, Cell Signalling) and Dapi (D1306, $1 \mu \mathrm{g} \mathrm{ml}^{-1}$, Life Technologies).

For western blotting: anti-Vegfr2 (9698, 1:2,000 working solution, Cell Signalling), anti-pVegfr2 (2478, 1:2,000 working solution, Cell Signalling), anti-pAKT (4060, 1:1,000 working solution, Cell Signalling), anti-AKT (4060, 1:1,000 working solution, Cell Signalling), anti-p-p44/42 mitogen-activated protein kinase (phospho-ERK, 9106, 1:2,000 working solution, Cell Signalling), anti-p44/42 mitogen-activated protein kinase (total ERK, 9102, 1:2,000 working solution, Cell Signalling), anti-pFOXO1 (9464, 1:1,000 working solution, Cell Signalling), anti-FOXO1 (2880, 1:1,000 working solution, Cell Signalling), anti-pPTEN (9549, 1:1,000 working solution, Cell Signalling) and anti-PTEN (9188, 1:1,000 working solution, Cell Signaling). Appropriate secondary antibodies were fluorescently labelled (Alexa Fluor donkey anti-rabbit, Alexa Fluor donkey anti-goat, Alexa Fluor donkey anti-rat, 1:400 working solution, Invitrogen) or conjugated to horseradish peroxidase (Anti-Rabbit IgG $(\mathrm{H}+\mathrm{L})$, 1:8,000 working solution, Vector Laboratories).

BMP9/10 blABs were from Genentech and PI3K inhibitors Wortmannin S2758 and Pictilisib S1065 were purchased from Selleckchem.

Fluorescence-activated cell sorting. Retinas were harvested at P5, dissected in cold PBS and digested in $1 \mathrm{mg} \mathrm{ml}^{-1}$ Collagenase type II (Sigma) in DMEM (Sigma). Cells were incubated with CD31-APC (BD 551262, $2 \mathrm{ng} \mathrm{ml}^{-1}$ ) and CD45-FITC (11-0452-85, $5 \mathrm{ng} \mathrm{ml}^{-1}$, eBioscience), Hoechst (B2261, $25 \mathrm{\mu g} \mathrm{ml}^{-1}$, Sigma Aldrich) and Pyronin Y (P9172, $200 \mathrm{ng} \mathrm{ml}^{-1}$, Sigma Aldrich). CD31 + I CD45 - endothelial cells were isolated by FACS and further analysed for their cell cycle distribution by two-dimensional analysis of Hoechst and Pyronin Y fluorescence signal.

mLECs isolation. MLECs were isolated from collagenase-digested lung tissue using rat-anti-mouse CD31 monoclonal antibody-coated Dynabeads (11035, Invitrogen). Primary mLECs were cultured in DMEM supplemented with $10 \%$ fetal bovine serum (FBS), $100 \mathrm{U} \mathrm{ml}^{-1}$ penicillin, $100 \mathrm{\mu g} \mathrm{ml}^{-1}$ streptomycin, $100 \mathrm{\mu g} \mathrm{ml}^{-1}$ endothelial cell mitogen (Biomedical Technologies, Inc.) and $10 \mathrm{U} \mathrm{ml}^{-1}$ heparin for 10 days.

Immunostaining. The eyes of P5/P6 pups were prefixed in 4\% PFA for 20 min at room temperature (rt). Retinas were dissected, blocked for $30 \mathrm{~min}$ at $\mathrm{rt}$ in blocking buffer ( $1 \%$ fetal bovine serum, 3\% BSA, $0.5 \%$ Triton X-100, $0.01 \% \mathrm{Na}$ deoxycholate, $0.02 \% \mathrm{Na}$ azide in PBS at pH 7.4) and then incubated with specific antibodies (mentioned above) in blocking buffer overnight. The following day retinas were washed and then incubated with IB4 and corresponding secondary antibody for $1 \mathrm{~h}$ at $\mathrm{rt}$ and mounted in fluorescent mounting medium (DAKO, Carpinteria, CA, USA).

Endothelial cells in culture were fixed in 4\% PFA for $15 \mathrm{~min}$, permeabilized in $0.1 \%$ Triton X-100 in PBS for 10 min, blocked in 3\% BSA (AB01088-00100, AmericanBIO) and incubated with the indicated primary antibodies overnight at $4{ }^{\circ} \mathrm{C}$ (mentioned above) followed by $1 \mathrm{~h}$ incubation with appropriate secondary antibodies at rt.

High-resolution pictures were acquired using a Leica SP5 confocal microscope with a Leica spectral detection system (Leica 15 SP detector) and the Leica application suite advanced fluorescence software. Quantification of retinal vasculature was done using ImageJ.

Cell culture and siRNA transfection. HUVEC cells were obtained from the Yale University Vascular Biology and Therapeutics Core Facility and cultured in EGM2Bullet kit medium (CC-3156 \& CC-4176, Lonza). Depletion of ALK1 was achieved by transfecting 25 pmol siRNA (Qiagen, mixture of S102659972 and S102758392) with Lipofectamine RNAiMax (Invitrogen), following the manufacturer's instructions. Transfection efficiency was assessed by western blotting and qPCR.

Figure 7 | PI3K inhibition rescues established retinal AVMs. (a) Schematic representation of the experimental strategy to assess Alk1 deletion or BMP9/10 blockade. Arrowhead indicates injection of $50 \mu \mathrm{g}$ Tx or BMP9/10 bIAB. (b) Quantification of AV shunt number 24h after Tx or BMP9/10 blAB administration. $n=8-10$ Retinas per group. Error bars: s.e.m. ${ }^{\star \star} P<0.01$ and ${ }^{\star \star \star} P<0.001$, Mann-Whitney $U$-test. (c-e) Ve-Cadherin and Erg1/2/3 staining of retinal flat mounts from $\mathrm{Ctrl}(\mathbf{c}), A / k 7^{i \Delta E C}$ - (d) and BMP9/10 bIAB- (e) treated P4 mice. Yellow arrowheads in d,e indicate AV shunts. Numbers of ERG1/2/3+ endothelial cell nuclei at branches of retinal veins and arteries are indicated in yellow. $(\mathbf{f}, \mathbf{g})$ Quantification of the number of nuclei at the connection of capillaries with veins (f) or arteries (g) in P4 and P5 retinal vessels. $n=6$ retinas per group. Error bars: s.e.m., ${ }^{\star \star} P<0.01$ and ${ }^{\star \star \star} P<0.001$, Mann-Whitney U-test. (h) Schematic representation of the experimental strategy to assess the effects of $\mathrm{PI} 3 \mathrm{~K}$ inhibition on $A / k 7^{\mathrm{i} \triangle \mathrm{EC}}$ vasculature $24 \mathrm{~h}$ post $\mathrm{Tx}$ injection. Arrowheads indicate the time course of Tx $(50 \mu \mathrm{g})$ and PI3Ki injections. (i,j) IB4 staining of retinal flat mounts from Alk $7^{\mathrm{i}} \mathrm{ECC}$ P5 mice treated with PBS (i) or PI3Ki $24 \mathrm{~h}$ post Tx injection (j). Yellow arrowheads in $\mathbf{i}, \mathbf{m}$ indicate AV shunts. (k) Quantification of AV shunt number, vascular density and number of branchpoints. $n=10-11$ Retinas per group. Error bars: s.e.m. ${ }^{\star \star} P<0.01$ and ${ }^{\star \star \star} P<0.001$, Mann-Whitney $U$-test. (I) Schematic representation of the experimental strategy to assess the effects of PI3K inhibition on the retinal vasculature $24 \mathrm{~h}$ post BMP9/10 bIAB-treated mice. Arrowheads indicate the time course of BMP9/10 bIAB and PI3Ki administration. $(\mathbf{m}, \mathbf{n})$ IB4 staining of retinal flat mounts from mice treated with BMP9/10 bIAB and $24 \mathrm{~h}$ later with PBS (m) or PI3Ki (n). Yellow arrowheads indicate AV shunts. (o) Quantification of AV shunt number, vascular density and number of branch points. $n=6$ Retinas per group. Error bars: s.e.m., ${ }^{\star} P<0.05$ and ${ }^{\star \star} P<0.01$, Mann-Whitney U-test. Scale bars, $100 \mu \mathrm{m}$ in $\mathbf{c}-\mathbf{e}, \mathbf{i}, \mathbf{j}, \mathbf{m}, \mathbf{n}$. a, artery in red; $\mathbf{v}$, vein in blue. 
a

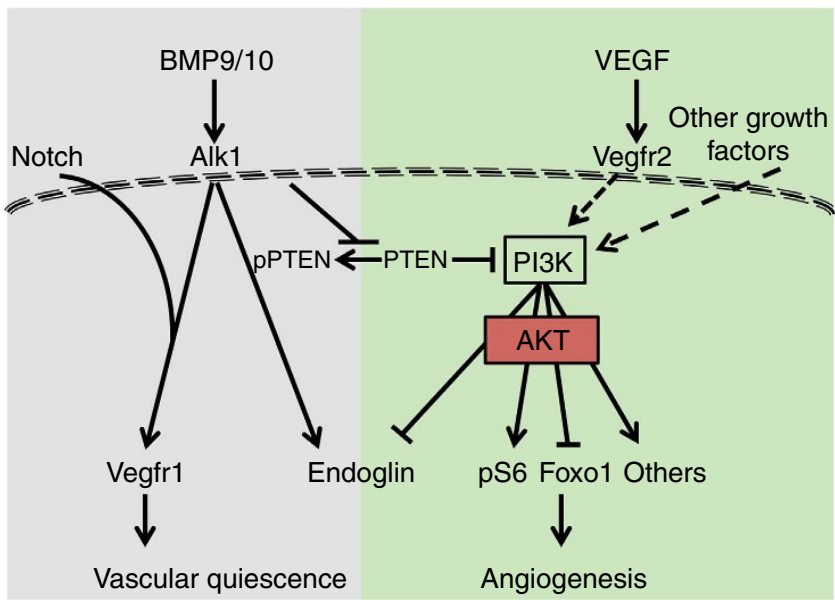

b

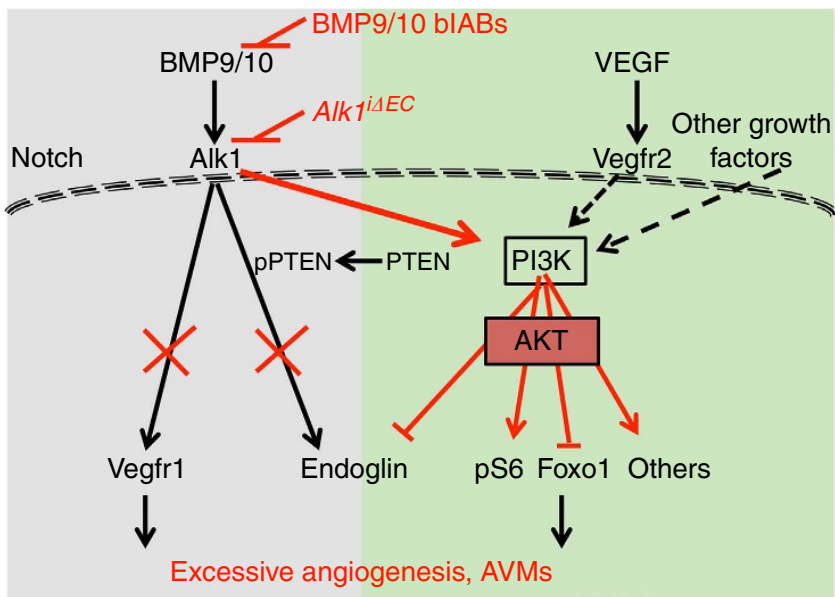

Figure 8 | Model for BMP9/10 signalling in maintenance of vascular quiescence. (a) BMP9/10 signalling through the Alk1 receptor in endothelial cells synergizes with Notch to induce expression of the antiangiogenic receptor -Vegfr 1 thereby repressing Vegf signalling and angiogenesis. Alk1 signalling also represses PI3K activation downstream of Vegfr2 and other growth factors, through inhibition of Pten expression and phosphorylation. PI3K promotes angiogenesis via Akt and downstream activation of ribosomal S6 (pS6), inhibition of Foxo1 and regulation of other downstream effectors. (b) Blocking BMP9/10-Alk1 signalling results in increased Pten expression and phosphorylation, and consequently in an excessive PI3K signalling, thereby inducing vascular defects. Blocking PI3K with PI3K inhibitors rescues excessive angiogenesis and vascular malformations in BMP signalling deficient mice.

Experiments were performed $48 \mathrm{~h}$ post transfection and results were compared with siRNA Ctrl (ON-TARGETplus Non-targeting Pool D-001810-10-05)

Western blotting. Cells were lysed with Laemmli buffer including phosphatase and protease inhibitors (Thermo Scientific, 78420, 1862209). Twenty micrograms of proteins were separated on $4-15 \%$ Criterion precast gel (567-1084, Bio-Rad) and transferred on nitrocellulose membrane (Biorad). Western blottings were developed with chemiluminescence horseradish peroxidase substrate (Millipore, WBKLS0500) on a Luminescent image Analyser, ImageQuant LAS $4000 \mathrm{mini}$ (GE Healthcare). Bands were quantified using ImageJ. See Supplementary Fig. 5 for the uncropped immunoblots.

Quantitative real-time PCR. RNAs from HUVEC or from mLECs were purified using RNeasy-kit (Qiagen). One RNA was reverse transcribed using SuperScript III (Invitrogen) and quantitative PCR were assayed using the corresponding primers (Qiagen): MmAcvrl1 (QT00161434), MmEng (QT00148981), MmUnc5b (QT00167846), MmFlt1 (QT00096292), MmNotch1 (QT00156982), MmJag1
(QT00115703), MmEfnb2 (QT00139202) and MmEphB4 (QT00120295). The expression levels were normalized to MmActin (QT00095242) and MmCdh5 (QT00110467).

Data availability. The authors declare that the data supporting the findings of this study are included within the article and its Supplementary Information files, or are available from the authors upon request.

\section{References}

1. Shovlin, C. L. Hereditary haemorrhagic telangiectasia: pathophysiology, diagnosis and treatment. Blood Rev. 24, 203-219 (2010).

2. McDonald, J. et al. Hereditary hemorrhagic telangiectasia: genetics and molecular diagnostics in a new era. Front. Genet. 6, 1 (2015).

3. McAllister, K. A. et al. Endoglin, a TGF-beta binding protein of endothelial cells, is the gene for hereditary haemorrhagic telangiectasia type 1. Nat. Genet 8, 345-351 (1994).

4. Johnson, D. W. et al. Mutations in the activin receptor-like kinase 1 gene in hereditary haemorrhagic telangiectasia type 2. Nat. Genet. 13, 189-195 (1996)

5. Gallione, C. J. et al. A combined syndrome of juvenile polyposis and hereditary haemorrhagic telangiectasia associated with mutations in MADH4 (SMAD4). Lancet 363, 852-859 (2004).

6. Oh, S. P. et al. Activin receptor-like kinase 1 modulates transforming growth factor-beta 1 signaling in the regulation of angiogenesis. Proc. Natl Acad. Sci. USA 97, 2626-2631 (2000).

7. Morrell, N. W. et al. Targeting BMP signalling in cardiovascular disease and anaemia. Nat. Rev. Cardiol. 13, 106-120 (2016).

8. David, L., Mallet, C., Mazerbourg, S., Feige, J. J. \& Bailly, S. Identification of BMP9 and BMP10 as functional activators of the orphan activin receptor-like kinase 1 (ALK1) in endothelial cells. Blood 109, 1953-1961 (2007).

9. Lebrin, F. et al. Endoglin promotes endothelial cell proliferation and TGFbeta/ALK1 signal transduction. EMBO J. 23, 4018-4028 (2004).

10. Bourdeau, A., Dumont, D. J. \& Letarte, M. A murine model of hereditary hemorrhagic telangiectasia. J. Clin. Invest. 104, 1343-1351 (1999).

11. Srinivasan, S. et al. A mouse model for hereditary hemorrhagic telangiectasia (HHT) type 2. Hum. Mol. Genet. 12, 473-482 (2003).

12. Roman, B. L. et al. Disruption of acvrl1 increases endothelial cell number in zebrafish cranial vessels. Development 129, 3009-3019 (2002).

13. Mahmoud, M. et al. Pathogenesis of arteriovenous malformations in the absence of endoglin. Circ. Res. 106, 1425-1433 (2010).

14. Park, S. O. et al. Real-time imaging of de novo arteriovenous malformation in mouse model of hereditary hemorrhagic telangiectasia. J. Clin. Invest. 119, 3487-3496 (2009).

15. Tual-Chalot, S. et al. Endothelial depletion of Acvrl1 in mice leads to arteriovenous malformations associated with reduced endoglin expression. PLoS ONE 9, e98646 (2014).

16. Garrido-Martin, E. M. et al. Common and distinctive pathogenetic features of arteriovenous malformations in hereditary hemorrhagic telangiectasia 1 and hereditary hemorrhagic telangiectasia 2 animal models-brief report. Arterioscler. Thromb. Vasc. Biol. 34, 2232-2236 (2014)

17. Wang, Y. et al. Ephrin-B2 controls VEGF-induced angiogenesis and lymphangiogenesis. Nature 465, 483-486 (2010).

18. Aspalter, I. M. et al. Alk1 and Alk5 inhibition by Nrp1 controls vascular sprouting downstream of Notch. Nat. Commun. 6, 7264 (2015).

19. Chen, H. et al. Context-dependent signaling defines roles of BMP9 and BMP10 in embryonic and postnatal development. Proc. Natl Acad. Sci. USA 110 11887-11892 (2013).

20. Koch, S. \& Claesson-Welsh, L. Signal transduction by vascular endothelial growth factor receptors. Cold Spring Harb. Perspect. Med. 2, a006502 (2012).

21. Baeyens, N. et al. Defective fluid shear stress mechanotransduction mediates hereditary hemorrhagic telangiectasia. J. Cell Biol. 214, 807-816 (2016).

22. Koch, A. W. et al. Robo4 maintains vessel integrity and inhibits angiogenesis by interacting with UNC5B. Dev. Cell 20, 33-46 (2011).

23. Atri, D., Larrivee, B., Eichmann, A. \& Simons, M. Endothelial signaling and the molecular basis of arteriovenous malformation. Cell Mol. Life Sci. 71, 867-883 (2013).

24. Haigh, J. J. et al. Cortical and retinal defects caused by dosage-dependent reductions in VEGF-A paracrine signaling. Dev. Biol. 262, 225-241 (2003).

25. Vazquez, F., Ramaswamy, S., Nakamura, N. \& Sellers, W. R. Phosphorylation of the PTEN tail regulates protein stability and function. Mol. Cell Biol. 20, 5010-5018 (2000).

26. Vazquez, F. et al. Phosphorylation of the PTEN tail acts as an inhibitory switch by preventing its recruitment into a protein complex. J. Biol. Chem. 276, 48627-48630 (2001).

27. Salih, D. A. \& Brunet, A. FoxO transcription factors in the maintenance of cellular homeostasis during aging. Curr. Opin. Cell Biol. 20, 126-136 (2008).

28. Serra, H. et al. PTEN mediates Notch-dependent stalk cell arrest in angiogenesis. Nat. Commun. 6, 7935 (2015). 
29. Wilhelm, K. et al. FOXO1 couples metabolic activity and growth state in the vascular endothelium. Nature 529, 216-220 (2016).

30. Dufner, A. \& Thomas, G. Ribosomal S6 kinase signaling and the control of translation. Exp. Cell Res. 253, 100-109 (1999).

31. Mouta-Bellum, C. et al. Organ-specific lymphangiectasia, arrested lymphatic sprouting, and maturation defects resulting from gene-targeting of the PI3K regulatory isoforms p85alpha, p55alpha, and p50alpha. Dev. Dyn. 238, 2670-2679 (2009).

32. Birdsey, G. M. et al. Transcription factor Erg regulates angiogenesis and endothelial apoptosis through VE-cadherin. Blood 111, 3498-3506 (2008).

33. Graupera, M. \& Potente, M. Regulation of angiogenesis by PI3K signaling networks. Exp. Cell Res. 319, 1348-1355 (2013).

34. Graupera, M. et al. Angiogenesis selectively requires the p110alpha isoform of PI3K to control endothelial cell migration. Nature 453, 662-666 (2008).

35. Lee, M. Y. et al. Endothelial Akt1 mediates angiogenesis by phosphorylating multiple angiogenic substrates. Proc. Natl Acad. Sci. USA 111, 12865-12870 (2014).

36. Rochon, E. R., Menon, P. G. \& Roman, B. L. Alk1 controls arterial endothelial cell migration in lumenized vessels. Development 143, 2593-2602 (2016).

37. Huang, J. et al. The role of COX-2 in mediating the effect of PTEN on BMP9 induced osteogenic differentiation in mouse embryonic fibroblasts. Biomaterials 35, 9649-9659 (2014).

38. Rahdar, M. et al. A phosphorylation-dependent intramolecular interaction regulates the membrane association and activity of the tumor suppressor PTEN. Proc. Natl Acad. Sci. USA 106, 480-485 (2009).

39. Tan, W. H. et al. The spectrum of vascular anomalies in patients with PTEN mutations: implications for diagnosis and management. J. Med. Genet. 44, 594-602 (2007)

40. Phung, T. L. et al. Pathological angiogenesis is induced by sustained Akt signaling and inhibited by rapamycin. Cancer Cell 10, 159-170 (2006).

41. Limaye, N. et al. Somatic mutations in angiopoietin receptor gene TEK cause solitary and multiple sporadic venous malformations. Nat. Genet. 41, 118-124 (2009).

42. Limaye, N. et al. Somatic activating PIK3CA mutations cause venous malformation. Am. J. Hum. Genet. 97, 914-921 (2015).

43. Tsuji-Tamura, K. \& Ogawa, M. Inhibition of the PI3K-Akt and mTORC1 signaling pathways promotes the elongation of vascular endothelial cells. J. Cell Sci. 129, 1165-1178 (2016).

44. Cirulli, A. et al. Vascular endothelial growth factor serum levels are elevated in patients with hereditary hemorrhagic telangiectasia. Acta Haematol. 110, 29-32 (2003).

45. Larrivee, B. et al. ALK1 signaling inhibits angiogenesis by cooperating with the Notch pathway. Dev. Cell 22, 489-500 (2012).

46. Moya, I. M. et al. Stalk cell phenotype depends on integration of Notch and Smad1/5 signaling cascades. Dev. Cell 22, 501-514 (2012).

47. Ricard, N. et al. BMP9 and BMP10 are critical for postnatal retinal vascular remodeling. Blood 119, 6162-6171 (2012).

48. Morikawa, M. et al. ChIP-seq reveals cell type-specific binding patterns of BMP-specific Smads and a novel binding motif. Nucleic Acids Res. 39, 8712-8727 (2011)

49. Taylor, K. L., Henderson, A. M. \& Hughes, C. C. Notch activation during endothelial cell network formation in vitro targets the basic HLH transcription factor HESR-1 and downregulates VEGFR-2/KDR expression. Microvasc. Res. 64, 372-383 (2002)

50. Han, C. et al. VEGF neutralization can prevent and normalize arteriovenous malformations in an animal model for hereditary hemorrhagic telangiectasia 2 . Angiogenesis 17, 823-830 (2014)
51. Bose, P., Holter, J. L. \& Selby, G. B. Bevacizumab in hereditary hemorrhagic telangiectasia. N. Engl. J. Med. 360, 2143-2144 (2009).

52. Oosting, S., Nagengast, W. \& de Vries, E. More on bevacizumab in hereditary hemorrhagic telangiectasia. N. Engl. J. Med. 361, 931-932 (2009).

53. Bose, P., Holter, J. L. \& Selby, G. B. Bevacizumab in hereditary hemorrhagic telangiectasia. N. Engl. J. Med. 360, 2143-2144 (2009).

54. Zarkada, G., Heinolainen, K., Makinen, T., Kubota, Y. \& Alitalo, K. VEGFR3 does not sustain retinal angiogenesis without VEGFR2. Proc. Natl Acad. Sci. USA 112, 761-766 (2015).

55. Simons, M. et al. State-of-the-art methods for evaluation of angiogenesis and tissue vascularization: a scientific statement from the American Heart Association. Circ. Res. 116, e99-e132 (2015).

56. Bernabeu, M. O. et al. Computer simulations reveal complex distribution of haemodynamic forces in a mouse retina model of angiogenesis. J. R. Soc. Interface 11, 1-17 (2014).

57. Corti, P. et al. Interaction between alk1 and blood flow in the development of arteriovenous malformations. Development 138, 1573-1582 (2011).

\section{Acknowledgements}

We thank Ralf Adams for Cdh5-CreERT2 mice. This project was supported by grants from NHLBI (1R01HLI125811) and NEI (1R01EY025979-01). R.O. and A.D. were supported by AHA postdoctoral fellowships 15POST25560114 and 14POST20380207, respectively.

\section{Author contributions}

R.O. provided experimental design, acquired and analysed data, drafted the manuscript. A.D., J.R.K., J.H., J.S.F., F.Z., B.L., M.L., A.A.U. and G.G. acquired data. K.K.H., W.C.S., F.V.C., M.G., M.Y., L.H.Y. and S.P.O. provided essential reagents. A.E. provided experimental design, analysed data and drafted the manuscript.

\section{Additional information}

Supplementary Information accompanies this paper at http://www.nature.com/ naturecommunications

Competing financial interests: The authors declare no competing financial interests.

Reprints and permission information is available online at http://npg.nature.com/ reprintsandpermissions/

How to cite this article: Ola, R. et al. PI3 kinase inhibition improves vascular malformations in mouse models of hereditary haemorrhagic telangiectasia. Nat. Commun. 7, 13650 doi: 10.1038/ncomms13650 (2016).

Publisher's note: Springer Nature remains neutral with regard to jurisdictional claims in published maps and institutional affiliations.

This work is licensed under a Creative Commons Attribution 4.0 International License. The images or other third party material in this article are included in the article's Creative Commons license, unless indicated otherwise in the credit line; if the material is not included under the Creative Commons license, users will need to obtain permission from the license holder to reproduce the material. To view a copy of this license, visit http://creativecommons.org/licenses/by/4.0/

(C) The Author(s) 2016 